\title{
Tetramic acids and derivatives by telluride-triggered Dieckmann cyclizations $^{1}$
}

\author{
Donald C. Dittmer,* Dmitry V. Avilov, Venkata Subbarao Kandula, Matthew T. Purzycki, \\ Zachary J. Martens, Elliot B. Hohn, and Matthew W. Bacler \\ Department of Chemistry, Room 1-014 CST, Syracuse University, Syracuse, NY 13244 \\ E-mail: dcdittme@syr.edu
}

Dedicated to Franklin A. Davis on the occasion of his $70^{\text {th }}$ anniversary

\begin{abstract}
Treatment of $\alpha$-bromoacyl amides of esters of $N$-protected $\alpha$-amino acids with lithium telluride yields an amide enolate which cyclizes to unstable tetramic acids (2, 4-pyrrolidinediones) which can be converted to stable derivatives (e.g. enol esters, silyl enol ethers, enol tosylates). Reaction conditions are modified to reduce unwanted side reactions: protonation of the enolate, selfcatalyzed intermolecular aldol rections of the tetramic acids, and potential racemization at the $\alpha$ carbon atom of the amino acid.
\end{abstract}

Keywords: Tetramic acids, lithium telluride, $\alpha$-bromoacyl amino acid esters, crown ether, racemization of silyl enol ethers, enol tosylates

\section{Introduction}

Tetramic acids (2, 4-pyrrolidinediones) are structural components of a number of natural products that show antibiotic, anticancer (cytotoxicity), fungicidal, and antiviral activity. ${ }^{2} \mathrm{~N}-$ Acylhomoserine lactones are used by some bacteria to exchange information ("quorum sensing"); one lactone degrades to a tetramic acid derivative that is a potent poison for certain encroaching microorganisms. ${ }^{3}$

Various methods have been used to prepare tetramic acids. ${ }^{2}$ The Dieckmann cyclization ${ }^{4 a}$ of $\mathrm{N}$-acyl- $\alpha$-amino esters and related methods (e.g. the Reformatsky reaction) ${ }^{4 \mathrm{~b}}$ are among the most useful. Enzyme-catalyzed formation of tetramic acids found in certain fungi proceeds by a Dieckmann cyclization. ${ }^{4 \mathrm{c}, 4 \mathrm{~d}}$ Racemization at the chiral $\alpha$-carbon atom of the starting amino acid ester caused by the base used in the Dieckmann reaction is an undesirable side reaction. ${ }^{5}$ Shorter reaction times (5-90 $\mathrm{min}$ ), lower temperatures (i.e. to room temperature), and the use of Dieckmann substrates (1,3-dicarbonyl and related compounds) that provide a more acidic proton 
than the $\alpha$-amino acid proton reduce or eliminate the amount of racemization. ${ }^{6-11}$ Bases such as fluoride ion (tetra- $n$-butylammonium fluoride, THF) and potassium $t$-butoxide are favorable, ${ }^{7}$ and the use of triethylamine in $\mathrm{CH}_{2} \mathrm{Cl}_{2}$-DMF has been successful in avoiding racemization. ${ }^{9}$ Neat benzene (reflux, $10 \mathrm{~h}$ ) is superior to alcoholic solvents in suppressing racemization by the methoxide ion. ${ }^{12}$ Solid phase syntheses of tetramic acids also show reduced racemization. ${ }^{10,13}$ Tetramic acids are not very stable and undergo self-catalyzed aldol condensations; they slowly undergo racemization both neat and in solution apparently involving acid catalysis by the C-3 protons. $^{12 \mathrm{a}, 13 \mathrm{c}, 14-16}$

The formation of a free enolate which undergoes the Dieckmann reaction by the action of a telluride dianion with $\alpha$-halocarbonyl compounds is advantageous because no strong base is involved; and the enolate anion is formed rapidly, irreversibly, and in high concentration that facilitates both inter- and intramolecular reactions. Analogy is made with the Reformatsky reaction, but with a telluride ion a carbon-tellurium intermediate is usually not involved. Elemental tellurium is produced and can be recovered and reused by reduction to a telluride ion unlike the usual Reformatsky reactions (e.g. with metals), which yield metal salts as waste. The telluride-induced synthesis of enolates from $\alpha$-halocarbonyl compounds can be termed a "nucleophilic reduction" since the halocarbonyl compound is reduced via the strongly nucleophilic telluride ion which is oxidized to elemental tellurium during the course of the reaction. The first example with a telluride ion was observed in 1957 during an attempt to prepare a telluride or ditelluride from sodium chloroacetate. ${ }^{17}$ The observed formation of acetic acid (from sodium acetate) may be interpreted as in Scheme 1.

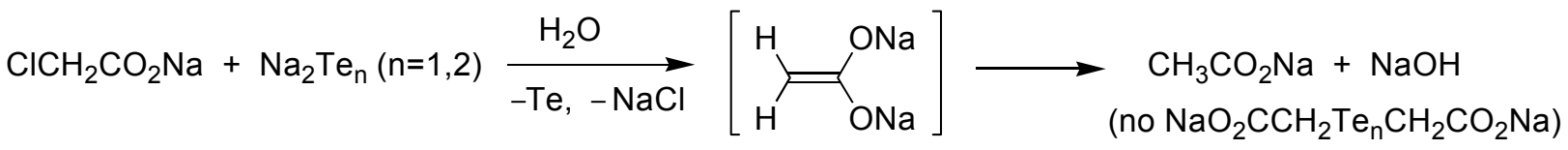

Scheme 1. "Nucleophilic reduction" of sodium chloroacetate. ${ }^{17}$

Investigators of telluride-induced enolate formation have reported protonations, ketene eliminations, intermolecular reactions with aldehydes and ketones, and intramolecular cyclizations involving esters and one aldehyde. ${ }^{18-22}$

\section{Results and Discussion}

Treatment of $\alpha$-amino acid derivatives 1 with an $\alpha$-bromoacyl bromide gives $\alpha$-bromoamides 2 (Table 1) (the NMR spectra show the presence of amide rotamers previously demonstrated ${ }^{21}$ ) which are treated with a slurry of lithium or sodium telluride in THF at room temperature. Preliminary results indicate that $\alpha$-chloroamides also can be used. A rapid precipitation of black, elemental tellurium heralds the irreversible formation of the amide enolate confirmed by 
deuteration of enolate 3 to give $\mathbf{4 b}$ (Scheme 2), confirmed by NMR of the crude reaction mixture. Cyclization of the enolate via the usual tetrahedral intermediate $\mathbf{5}$ yields tetramic acids $\mathbf{6}$ (Scheme 2) (Table 2).
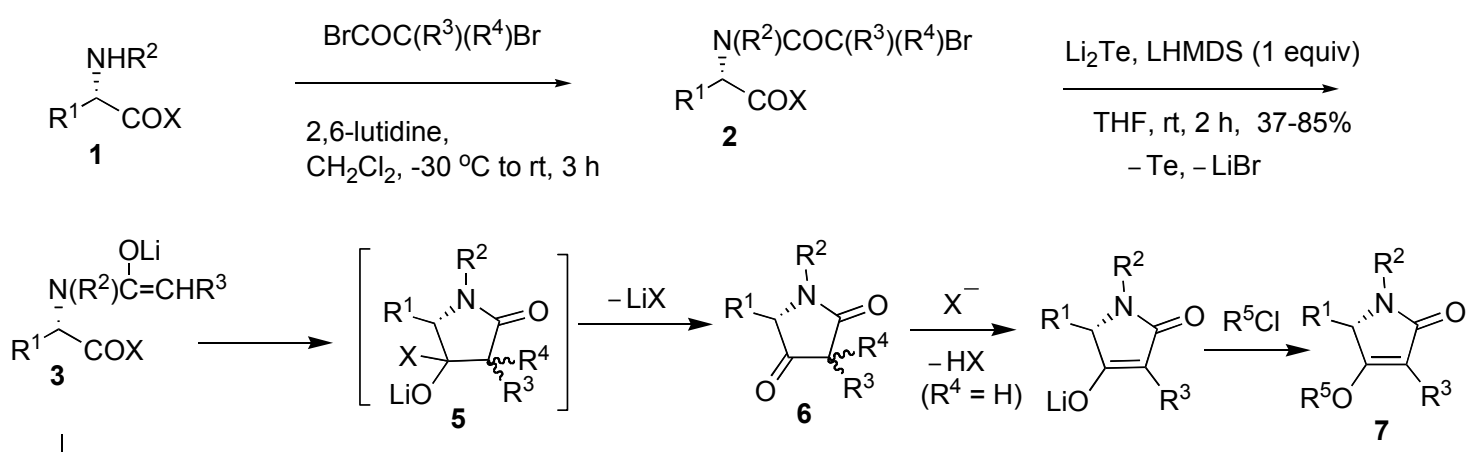

$$
\begin{aligned}
& \text { protonation }
\end{aligned}
$$

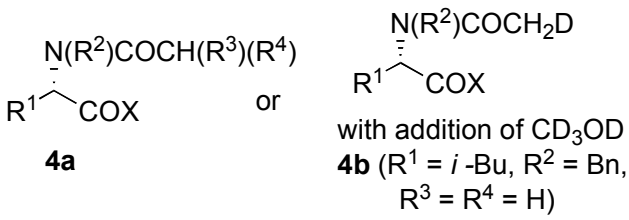

Scheme 2. Tetramic acids 6 and derivatives 7 of their enolates from $N$ - $\alpha$-bromoacyl derivatives 2 of $\alpha$-amino acid esters or a Weinreb amide $\mathbf{2 h}$ and telluride ion.

Table 1. Bromoamides of $\alpha$-amino acid derivatives

\begin{tabular}{ccccccc}
\hline $\mathbf{2}$ & $\mathrm{R}^{1}$ & $\mathrm{R}^{2}$ & $\mathrm{R}^{3}$ & $\mathrm{R}^{4}$ & X & Yield, \% \\
\hline $\mathbf{a}$ & $\mathrm{Bn}$ & $\mathrm{Bn}$ & $\mathrm{H}$ & $\mathrm{H}$ & $\mathrm{OMe}$ & 97 \\
$\mathbf{b}$ & $i-\mathrm{Bu}$ & $\mathrm{Bn}$ & $\mathrm{H}$ & $\mathrm{H}$ & $\mathrm{OMe}$ & 97 \\
$\mathbf{c}$ & $\mathrm{Bn}$ & $\mathrm{PMB}$ & $\mathrm{H}$ & $\mathrm{H}$ & $\mathrm{OMe}$ & 94 \\
$\mathbf{d}$ & $i-\mathrm{Bu}$ & $\mathrm{PMB}$ & $\mathrm{H}$ & $\mathrm{H}$ & $\mathrm{OMe}$ & 97 \\
$\mathbf{e}$ & $s-\mathrm{Bu}$ & $\mathrm{PMB}$ & $\mathrm{H}$ & $\mathrm{H}$ & $\mathrm{OMe}$ & 91 \\
$\mathbf{f}$ & $s-\mathrm{Bu}$ & $\mathrm{DMB}$ & $\mathrm{H}$ & $\mathrm{H}$ & $\mathrm{OMe}$ & 96 \\
$\mathbf{g}$ & $s-\mathrm{Bu}$ & $\mathrm{Bn}$ & $\mathrm{H}$ & $\mathrm{H}$ & $\mathrm{OMe}$ & 100 \\
$\mathbf{h}^{\mathrm{a}}$ & \multicolumn{2}{c}{$\left(\mathrm{CH}_{2}\right)_{3}{ }^{-}$} & $\mathrm{H}$ & $\mathrm{H}$ & $\mathrm{N}(\mathrm{Me})(\mathrm{OMe})$ & 71 \\
$\mathbf{i}$ & $\mathrm{Bn}$ & $\mathrm{Bn}$ & $\mathrm{CH}_{3}$ & $\mathrm{H}$ & $\mathrm{OMe}$ & 97 \\
$\mathbf{j}$ & $i-\mathrm{Bu}$ & $\mathrm{Bn}$ & $\mathrm{CH}_{3}$ & $\mathrm{H}$ & $\mathrm{OMe}$ & 97 \\
$\mathbf{k}$ & $i-\mathrm{Bu}$ & $\mathrm{Bn}$ & $\mathrm{CH}_{3}$ & $\mathrm{CH}_{3}$ & $\mathrm{OMe}$ & 99 \\
\hline
\end{tabular}

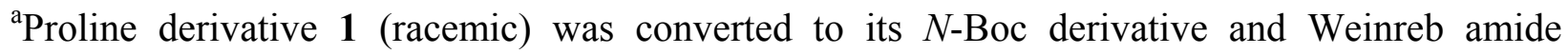
followed by removal of the Boc group by treatment with trifluoroacetic acid before conversion to 2h. 
Table 2. Tetramic acids $6^{\mathrm{a}}$

\begin{tabular}{cccccc}
\hline $\mathbf{6}$ & $\mathrm{R}^{1}$ & $\mathrm{R}^{2}$ & $\mathrm{R}^{3}$ & $\mathrm{R}^{4}$ & Yield, \% \\
\hline $\mathbf{c}$ & $\mathrm{Bn}$ & $\mathrm{PMB}$ & $\mathrm{H}$ & $\mathrm{H}$ & 62 (crude) \\
$\mathbf{e}$ & $s-\mathrm{Bu}$ & $\mathrm{PMB}$ & $\mathrm{H}$ & $\mathrm{H}$ & 65 \\
$\mathbf{h}$ & \multicolumn{2}{c}{${ }^{-\left(\mathrm{CH}_{2}\right)_{3^{-}}}$} & $\mathrm{H}$ & $\mathrm{H}$ & 80 \\
$\mathbf{k}$ & $i-\mathrm{Bu}$ & $\mathrm{Bn}$ & $\mathrm{Me}$ & $\mathrm{Me}$ & 79 \\
\hline
\end{tabular}

${ }^{\mathrm{a}}$ Most of the other tetramic acids were unstable and were converted to derivatives.

Table 3. Derivatives 7 of enolates of tetramic acids

\begin{tabular}{cccccc}
\hline $\mathbf{7}$ & $\mathrm{R}^{1}$ & $\mathrm{R}^{2}$ & $\mathrm{R}^{3}$ & $\mathrm{R}^{5}$ & Yield, \% \\
\hline $\mathbf{a}$ & $\mathrm{Bn}$ & $\mathrm{Bn}$ & $\mathrm{H}$ & $\mathrm{TBDPS}$ & $55^{\mathrm{a}}$ \\
$\mathbf{b}$ & $i-\mathrm{Bu}$ & $\mathrm{Bn}$ & $\mathrm{H}$ & TBDPS & $82^{\mathrm{a}}$ \\
$\mathbf{e}$ & $s-\mathrm{Bu}$ & $\mathrm{PMB}$ & $\mathrm{H}$ & $\mathrm{Ts}$ & 85 \\
$\mathbf{f}$ & $s-\mathrm{Bu}$ & $\mathrm{DMB}$ & $\mathrm{H}$ & $\mathrm{PhCH}_{2} \mathrm{CO}$ & 73 \\
$\mathbf{g}$ & $s-\mathrm{Bu}$ & $\mathrm{Bn}$ & $\mathrm{H}$ & $\mathrm{PhCO}$ & 73 \\
$\mathbf{i}$ & $\mathrm{Bn}$ & $\mathrm{Bn}$ & $\mathrm{CH}_{3}$ & $\mathrm{TBDPS}$ & 77 \\
$\mathbf{j}$ & $i-\mathrm{Bu}$ & $\mathrm{Bn}$ & $\mathrm{CH}_{3}$ & TBDPS & 95 \\
\hline
\end{tabular}

${ }^{a}$ Yield was $92 \%$ for $\mathbf{7 a}$ and $100 \%$ for $\mathbf{7 b}$ when 3.5 equivalents of $t$-butyldiphenylsilyl chloride (TBDPSCl) were used, but the product was racemized.

Because of the instability of tetramic acids only three $6 \mathbf{e}, \mathbf{6 h}$, and $6 \mathbf{k}$ were isolated. The rest except 6c were converted to stable $t$-butyldiphenylsilyl ethers (TBDPS), enol esters, or enol tosylates 7a-b, 7e-g, 7i-j (Scheme 2) (Table 3). Once the lithium or sodium enolate is formed, it is susceptible to protonation to give the amide, $\mathbf{4 a}$, which halts tetramic acid formation. To control this undesired protonation, potential proton donors such as the amino-acid-derived $\mathrm{NH}$ proton and acidic products (tetramic acid, methanol) must be nullified. This can be accomplished by the use of an $\mathrm{N}$-protected amino acid and by neutralization of acids with an equivalent of base. The protection of the NH group of the amino acid starting materials by benzyl (Bn), $p$ methoxybenzyl (PMB), or 3, 4-dimethoxybenzyl (DMB) groups is accomplished by treatment with the aryl aldehyde to give the imine which is reduced with sodium borohydride. ${ }^{23}$ While an added base can reduce protonation, it may also cause racemization at the $\alpha$-carbon of the former amino acid. For the telluride process shown in Scheme 2 to be most useful it must proceed between the Scylla of "protonation" to $\mathbf{4 a}$ and the Charybdis of racemization to racemic $6 .{ }^{24}$

Reduction of the 4-carbonyl group of tetramic acid $\mathbf{6 c}$ is accomplished readily with sodium borohydride. $^{5 b, 25}$ The resulting diastereomer $\mathbf{8}$ derived from $\mathbf{6 c}$ is obtained with a $d r$ 20:1, ${ }^{25}$ which indicates little racemization at C-5 (Scheme 3). The diastereomeric ratio was determined using ${ }^{1} \mathrm{H}$ NMR spectroscopy and was based on known literature data. ${ }^{25}$ 

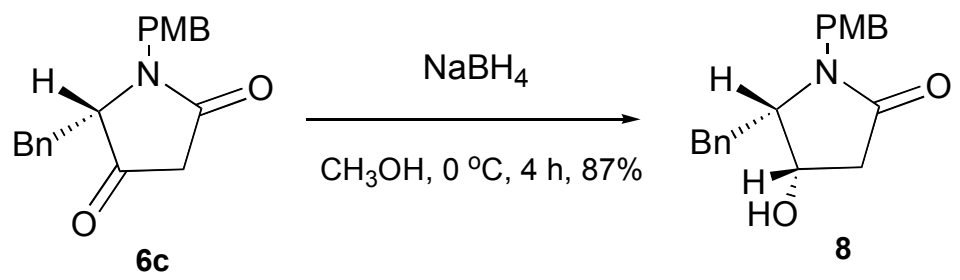

Scheme 3. Reduction of the 4-carbonyl group of tetramic acids.

Weinreb amides ${ }^{26}$ were investigated (Scheme 2, 2h) because they are less acidifying for the $\alpha$-amino acid proton, and the tetrahedral intermediate $\mathbf{5}$ is more stable than that derived from the methyl ester. ${ }^{25 c}$ This latter effect delays the formation of the acidifying C-4 carbonyl group which is expected to reduce both "back-protonation" and racemization as well as potentially limiting self-catalyzed reactions of the tetramic acids. While the bromoacetylation of the proline Weinreb amide was successful, that of $N$-benzyl leucine Weinreb amide was not, starting material being recovered. An interaction of bromoacetyl bromide with the Weinreb amide may provide an unstable intermediate that transfers the acyl group to the nitrogen atom of the amino acid derivative. This is reminiscent of the intermediate derived from DMF in its catalysis of acid chloride and formate ester formation in which there is acyl transfer. ${ }^{27}$ The more rigid proline framework lacks the $N$-benzyl group and may allow more favorable conformations, $\mathbf{1 0} v s \mathbf{9}$, for acyl transfer.
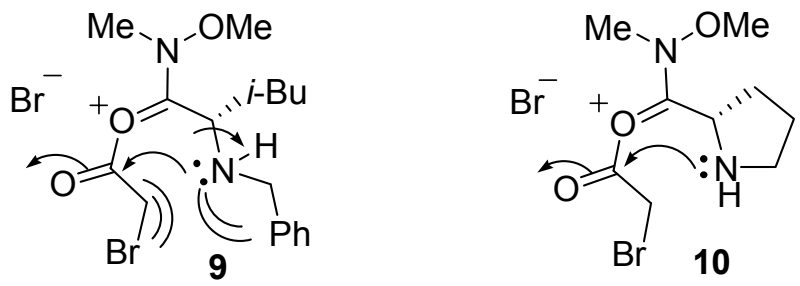

Best yields of the tetramic acid $\mathbf{6 h}$ derived from racemic proline were obtained when an equivalent of LHMDS or LDA was added. The bases LDA and NaH (heterogeneous in THF) are reported to cause racemization of tetramic acids ${ }^{25 c, 28}$ and were not investigated further. Other conditions involving methyl esters of $N$-bromoacetyl derivatives of $N$-benzylamino acids produced significant amounts of the protonated enolate $4 \mathbf{a}$ : higher temperature $\left(65-70{ }^{\circ} \mathrm{C}\right)$; longer reaction time (5 days); different solvents (9:1 ether-THF, DME); additives [TiCl $4, \mathrm{LiOTf}_{\text {, }}$ $\mathrm{In}(\mathrm{OTf})_{3}, \mathrm{Sc}(\mathrm{OTf})_{3}, \mathrm{Yb}(\mathrm{OTf})_{3}, \mathrm{ZnBr}_{2}, \mathrm{MgBr}_{2}, 4 \mathrm{~A}$ molecular sieves; DBU]; 0.5 equiv. excess of $\mathrm{LiEt}_{3} \mathrm{BH}$. Only with DME at $65-70{ }^{\circ} \mathrm{C}$ and with DBU (base effect) were the amounts of tetramic acid 6 greater than $\mathbf{4 a}$ (ratios $\mathbf{6} / \mathbf{4 a}=54 / 46,56 / 44$, respectively). Application of the telluride procedure to the synthesis of 3,3-disubstituted-tetramic acids is not complicated by "back protonation" or a self-catalyzed aldol condensation because the acidic protons are not present. No extra base is required. The 3,3-dimethyltetramic acid $\mathbf{6 k}$ derived from $(L)$-leucine is the sole product although its formation is slower (Scheme 4). 


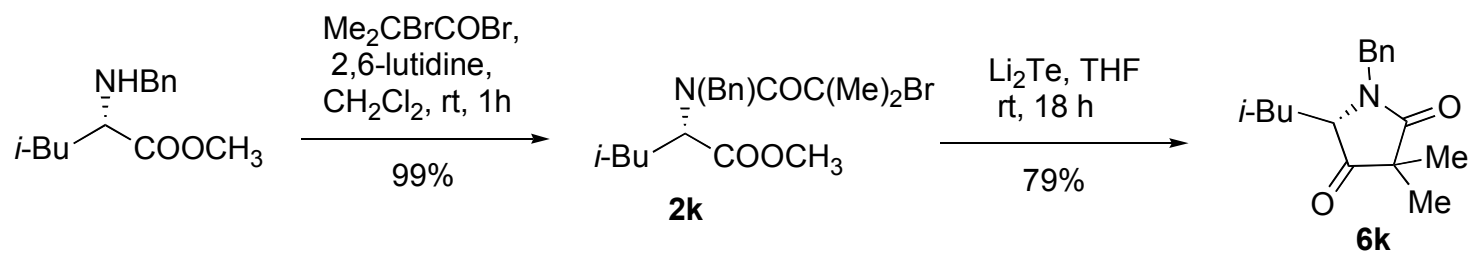

Scheme 4. Synthesis of 3,3-dimethyltetramic acid $\mathbf{6 k}$.

The addition of one equivalent of crown ether (15-crown-5) to the sodium telluride-triggered reaction avoids the need for added base in the conversion of the $\alpha$-bromoacetamides of $L$ phenylalanine, $L$-leucine, and $L$-isoleucine methyl esters (Scheme 5) and reduces the reaction time from 40-50 min. to 20-30 min. Presumably, the crown ether facilitates the sodium enolate's attack on the carbonyl group of the ester in the Dieckmann reaction by formation of a more favorable conformation while shielding the enolate from protonation and/or by increasing the rate of deprotonation of the tetramic acid by the crown-complexed sodium methoxide and perhaps by hydrogen bonding of the product methanol with the oxygen atoms of the crown ether to slow its diffusion to the enolate anion. Figure 1 shows how 15 -crown-5 might affect an halophilic attack by sodium telluride.
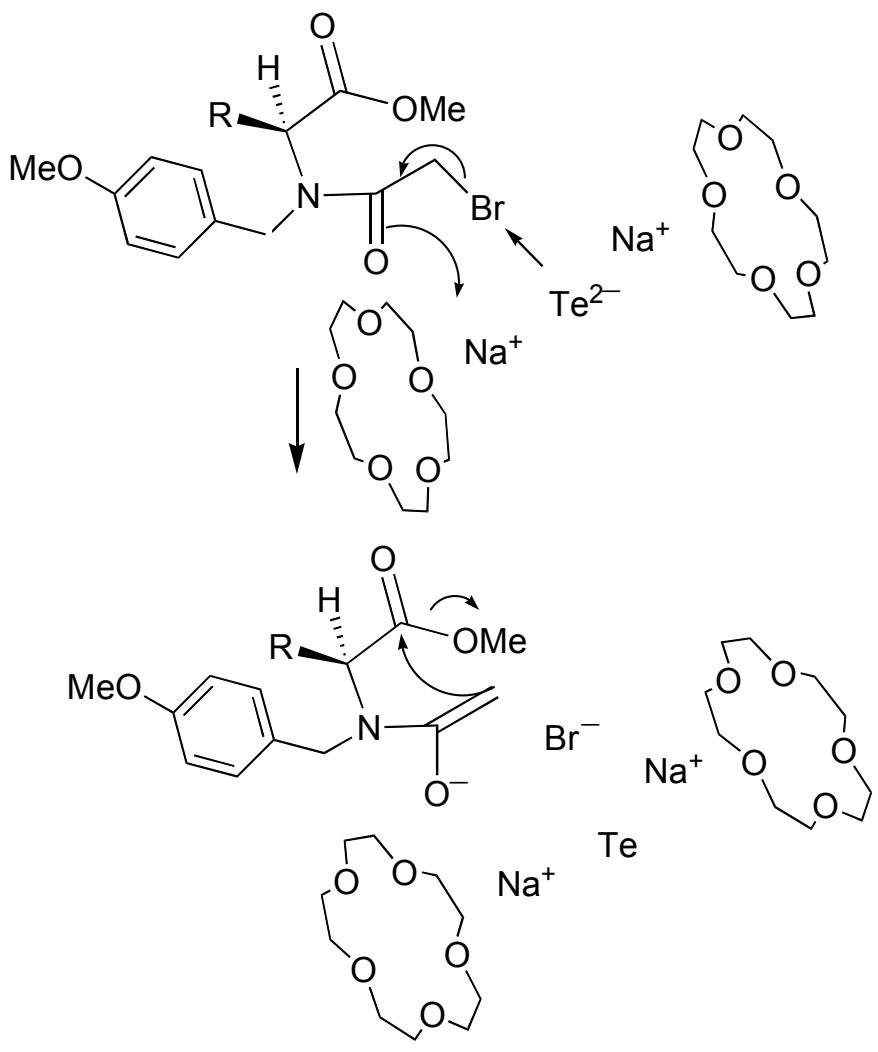

Figure 1. Crown ether effect. 
Crown ethers do not seem to have been used in aldol reactions involving oxazolidinone chiral auxiliaries, but catalysis by chiral crown ethers complexed with a Lewis acid (e.g. $\mathrm{Pb}^{2+}$, $\mathrm{Ce}^{3+}$ ) is observed in reactions of silyl enol ethers with aldehydes. ${ }^{29 a, b}$ In reactions of other silyl enol ethers with aldehydes catalyzed by gallium (III) triflate in water-ethanol, hydrolysis of the enol ether was complete and no aldol product was observed. In the presence of chiral semi-crown ethers, only traces of hydrolysis product were observed; the aldol product was obtained in yields of $49-90 \%$ with mainly syn selectivity. ${ }^{29 c}$ The chiral gallium-semi-crown ether catalysts are said to accelerate the aldol reaction relative to the hydrolysis reaction.

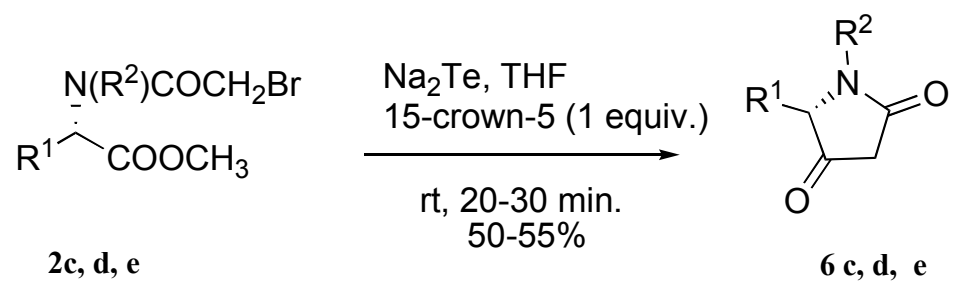

Scheme 5. Cyclization of $\alpha$-bromoamides by sodium telluride in the presence of a crown ether.

Application of the telluride procedure ( $\mathrm{Li}_{2} \mathrm{Te}, 1$ equiv. LHMDS, THF, $\mathrm{rt}$ ) for the synthesis of tetramic acids indicates that little racemization occurred at the C-5 position as determined by NMR studies on tetramic acid derivatives obtained from $L$-isoleucine [( $2 S, 3 S)$-2-amino-3methylpentanoic acid]. Tetramic acids derived from isoleucine have been used previously to determine the amount of racemization. ${ }^{5 b, 7}$ Because the formation of 3,3-dimethyltetramic acids (Scheme 4) does not require added base to suppress "back protonation", the danger of racemization by the base is avoided.

Conversion of tetramic acids to enol ethers, enol esters or enol tosylates (Scheme 2, Table 3) could circumvent the self acid-catalyzed aldol condensation and acid-catalyzed racemization and provide stable intermediates that could readily be converted back to the parent tetramic acid if that is desired. While the $t$-butyldiphenylsilyl (TBDPS) enol ethers are stable, their preparation resulted in some racemization, most likely caused by silylation of the amide carbonyl oxygen ${ }^{30}$ to give an intermediate (Scheme 6) in which the $\mathrm{CH}$ proton derived from the $\alpha$-amino acid is expected to have greater acidity. Workup removes the silyl group from the amide leaving racemized 4-silyl enol ether derivatives. Yields of the silyl enol ether were best (7a, 92\%, and $\mathbf{7 b}, 100 \%$ ) when 3.5 equivalents of TBDPSCl were used, but racemization of $7 \mathbf{a}$ was nearly complete, er $=53: 47$. It is likely that all the silyl enol ethers were racemized to some extent (small rotations for $7 \mathbf{a}$ and $\mathbf{7 b}$ ) when only 1.15 equivalents of TBDPSCl were used but this was not investigated further. 


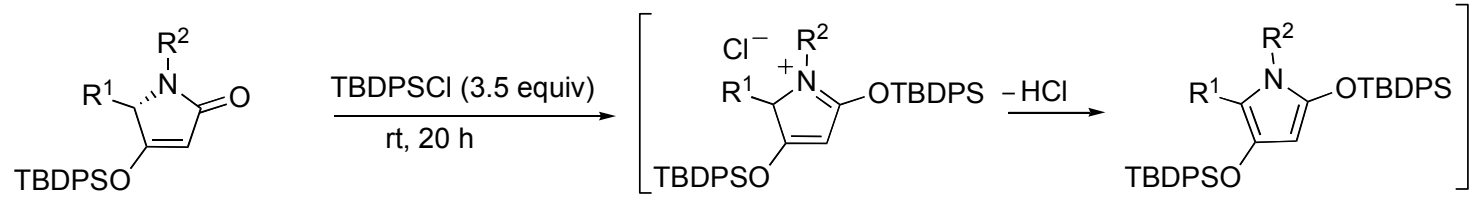

7a: $\mathrm{R}^{1}=\mathrm{Bn}, \mathrm{R}^{2}=\mathrm{Bn}$

7b: $\mathrm{R}^{1}=i-\mathrm{Bu}, \mathrm{R}^{2}=\mathrm{Bn}$

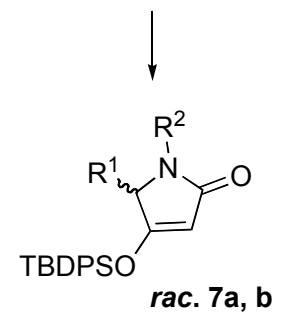

Scheme 6. Racemization of $t$-butyldiphenylsilyl enol ethers of tetramic acids.

The preparation of the enol benzoate $\mathbf{7 g}$ and tosylate $7 \mathbf{e}$ (Scheme 2, Table 3 ) derived from $L$ isoleucine did not involve racemization at the $\mathrm{C}-5$ of the tetramate derivative because the proton NMR spectra did not show the presence of the other diastereomer (which would be derived from $(2 R, 3 S)$-2-amino-3-methylpentanoic acid).

The $N$-protective groups may need to be removed from the tetramic acid derivatives for further synthesis operations. The $\mathrm{N}$-benzyl group could not be cleaved under frequently used conditions: $\mathrm{H}_{2}, \mathrm{Pd} / \mathrm{C}$, EtOAc or EtOH; $\mathrm{H}_{2}, \mathrm{Pd}(\mathrm{OH})_{2} / \mathrm{C}$; ceric ammonium nitrate (CAN); DDQ. Removal of an $N$-3,4-dimethoxybenzyl (3,4-DMB) group is accomplished by CAN but not by DDQ (Scheme 7). ${ }^{31}$
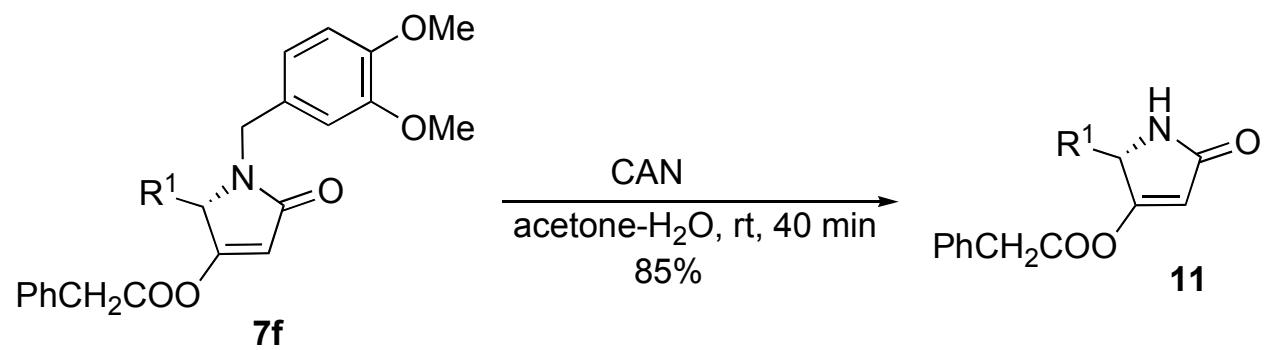

Scheme 7. Removal of an N-3,4-dimethoxybenzyl group.

\section{Conclusions}

Cyclization of $N$-protected $\alpha$-bromoacyl amides of $\alpha$-amino acid methyl esters or Weinreb amides to tetramic acids is initiated by telluride ion, which rapidly and irreversibly forms the amide enolate. Proton transfer from an unprotected NH group or from the tetramic acid or methanol products to the enolate anion inhibits the Dieckmann cyclization and reduces the yield. 
This undesirable protonation is avoided by the use of dry solvent, replacement of the NH group with an $N$-benzyl group, by addition of one equivalent of base (LHMDS) or, alternatively, one equivalent of a crown ether (15-crown-5) with an equivalent of sodium telluride. No added base or crown ether is needed to obtain a good yield of a 3,3-dimethyltetramic acid which has no acidic C-3 protons. Racemization is mainly avoided in the telluride process, but conversion of a tetramic acid to a stable $t$-butyldiphenylsilyl enol ether resulted in racemization. Enol benzoate and tosylate underwent no detectable epimerization at the $\alpha$-amino acid proton of the tetramate derived from $L$-isoleucine.

\section{Acknowledgements}

This research was supported by Syracuse University. Matthew Purzycki, Zachary Martens, Elliot Hohn, and Matthew Bacler were supported by the NSF-Research Experience for Undergraduates program. We thank Professor John Chisholm for the use of an HPLC instrument and Patrick Carberry for his help.

\section{Experimental Section}

General. NMR spectra were recorded on a Bruker DPX300 or DPX 500 instrument with tetramethylsilane as internal standard. Chemical shifts are reported in ppm downfield from tetramethylsilane. Optical rotations were measured on a JASCO 1000 or Rudolph Autopol III polarimeter. All reactions were conducted in an atmosphere of dry argon or nitrogen according to standard vacuum line techniques. Unless otherwise noted, all commercially available reagents were used as received without further purification. Tetrahydrofuran was distilled or vacuumtransferred from sodium or potassium benzophenone ketyl immediately before use; ether, from sodium benzophenone ketyl; and dichloromethane, from calcium hydride. Pyridine and triethylamine were distilled from sodium hydroxide immediately before use. All other liquid reagents were distilled immediately before use. Thin layer chromatography was performed with the use of Merck Silica Gel 60 plates impregnated with a fluorescent indicator $(254 \mathrm{~nm})$. Thin layer chromatography plates were visualized by exposure to ultraviolet light, iodine, anisaldehyde, or phosphomolybdic acid. Flash column chromatography was performed with the use of Premium Grade Silica Gel.

\section{General procedure for the preparation of lithium telluride ${ }^{21}$}

A solution of lithium triethylborohydride in THF $(7.98 \mathrm{~mL}, 1 \mathrm{M}, 7.98 \mathrm{mmol})$ was added to a suspension of tellurium pieces $(509 \mathrm{mg}, 3.99 \mathrm{mmol})$ in THF $(7.95 \mathrm{~mL})$ at room temperature. The mixture was stirred for $20 \mathrm{~h}$. Hydrogen evolved, and the color of the mixture gradually becomes nearly white. This $0.25 \mathrm{M}$ "stock suspension" of finely divided lithium telluride in THF is stable 
for weeks at room temperature under an argon atmosphere and the slurry can be transferred easily with an airtight syringe equipped with a stainless steel needle.

Preparation of N-protected-N-haloacylamino acid esters 2a-k (Procedure A). Bromoacetyl bromide $(1.36 \mathrm{~g}, 6.73 \mathrm{mmol})$ was added dropwise to a mixture of $N$-benzyl or $N$-PMB or $N$-3,4-DMB protected amino acid ester (1.32 g, $5.61 \mathrm{mmol})$ and 2,6-lutidine (0.90 g, $8.42 \mathrm{mmol})$ in $\mathrm{CH}_{2} \mathrm{Cl}_{2}(21.6 \mathrm{~mL})$ at room temperature. The mixture was stirred for $1 \mathrm{~h}$, poured into saturated aqueous $\mathrm{NH}_{4} \mathrm{Cl}(20 \mathrm{~mL})$, and extracted with EtOAc $(3 \times 10 \mathrm{~mL})$. The crude mixture was dried $\left(\mathrm{Na}_{2} \mathrm{SO}_{4}\right)$ and chromatographed on silica gel (hexanes/EtOAc 3:1) to give halo esters (71-100\%). Synthesis of tetramic acids $6 \mathrm{c}, 6 \mathrm{~d}, 6 \mathrm{e}$ in the presence of 15-crown-5 ether (Procedure B). A mixture of powdered Te ( 1 equiv.), Na chips ( 2 equiv.) and naphthalene ( 0.2 equiv) in anhydrous THF is refluxed under nitrogen until the mixture becomes a white suspension which is transferred to a stirred solution of $\mathbf{2 c}, \mathbf{2 d}$, or $\mathbf{2 e}(2.38 \mathrm{mmol})$ in dry THF. 15-Crown-5 ether (1 equiv.) was added and the reaction mixture was stirred at ambient temperature for 20-30 min., quenched with saturated aqueous $\mathrm{NH}_{4} \mathrm{Cl}(5 \mathrm{~mL})$, filtered through a celite pad, extracted with ethyl acetate, and dried $\left(\mathrm{Na}_{2} \mathrm{SO}_{4}\right)$. The crude tetramic acid (55-65\%) was used for further reactions such as the preparation of derivatives or for reduction of the C-4 carbonyl group. Freshly prepared $\mathrm{Na}_{2} \mathrm{Te}$ is recommended.

\section{Preparation of enol derivatives of tetramic acids ( $O$-silyl, $O$-tosyl, $O$-acetyl, $O$-benzoyl, $O$ - phenylacetyl tetramates)}

$\boldsymbol{O}$-Silyl tetramates 7a, 7b, 7i, 7j (Procedure C). Lithium telluride (5.38 mL, $1.35 \mathrm{mmol}, 0.25 \mathrm{M}$ suspension) was added to a solution of haloacylamino acid esters $\mathbf{2 a}, \mathbf{2 b}, \mathbf{2 i}, \mathbf{2} \mathbf{j}(0.47 \mathrm{~g}, 1.32$ $\mathrm{mmol})$ in THF $(12.7 \mathrm{~mL})$ followed by a THF solution of LHMDS (1.32 mL, $1.32 \mathrm{mmol}, 1 \mathrm{M})$ at room temperature. The mixture was stirred for $40 \mathrm{~min}$, and freshly distilled $t-\mathrm{BuPh}_{2} \mathrm{SiCl}(0.42 \mathrm{~g}$, $1.53 \mathrm{mmol}$ ) was added. The mixture was stirred for $20 \mathrm{~h}$, poured into saturated aqueous $\mathrm{NH}_{4} \mathrm{Cl}$ $(10 \mathrm{~mL})$, filtered through a celite pad to remove elemental tellurium, and extracted with ethyl acetate $(3 \times 20 \mathrm{~mL})$. The crude mixture was dried $\left(\mathrm{Na}_{2} \mathrm{SO}_{4}\right)$ and chromatographed on silica gel (hexane/EtOAc 3:1) to give $O$-silyl tetramates (55-95\%).

$O$-Phenylacetyl (7f, $\left.\mathrm{R}^{4}=\mathrm{PhCH}_{2} \mathrm{CO}\right)$, and $O$-benzoyl $\left(7 \mathrm{~g}, \mathrm{R}^{4}=\mathbf{P h C O}\right.$ ) tetramates (Procedure D). Lithium telluride $(2.55 \mathrm{~mL}, 0.64 \mathrm{mmol}, 0.25 \mathrm{M}$ suspension) was added to a solution of haloacylamino acid esters $\mathbf{2} \mathbf{i}, \mathbf{2 h}(0.22 \mathrm{~g}, 0.63 \mathrm{mmol})$ in THF $(6.3 \mathrm{~mL})$ followed by a THF solution of LHMDS $(0.64 \mathrm{~mL}, 0.64 \mathrm{mmol}, 1 \mathrm{M})$ at room temperature. The mixture was stirred for $20 \mathrm{~h}$, poured into $3 \%$ aq. $\mathrm{HCl}(5 \mathrm{~mL})$, and extracted with methylene chloride $(3 \times 5$ $\mathrm{mL})$. The organic layer was dried $\left(\mathrm{Na}_{2} \mathrm{SO}_{4}\right)$, the solvent was evaporated, and the residue was dried in a vacuum for $4 \mathrm{~h}$. The residue was dissolved in methylene chloride $(9.5 \mathrm{~mL})$; and triethylamine $(0.095 \mathrm{~g}, 0.94 \mathrm{mmol})$ was added, followed by the acid chloride $(0.10 \mathrm{~g}, 0.75 \mathrm{mmol})$ at room temperature. The mixture was stirred $(22 \mathrm{~h})$, poured into saturated aqueous $\mathrm{NH}_{4} \mathrm{Cl}(5$ $\mathrm{mL})$, and extracted with EtOAc $(3 \times 20 \mathrm{~mL})$. The crude mixture was dried $\left(\mathrm{Na}_{2} \mathrm{SO}_{4}\right)$ and chromatographed on silica gel (hexanes/EtOAc 3:1) to give $7 \mathbf{f}$ or $\mathbf{7 g}$. 
$\boldsymbol{O}$-Tosyl tetramate 7e (Procedure E). The procedure reported previously for an $N$-Boc tetramic acid was followed. ${ }^{32}$ Diisopropyl ethylamine $(0.5 \mathrm{~mL}, 2.9 \mathrm{mmol})$ and freshly recrystallized $p$ toluenesulfonyl chloride $(0.27 \mathrm{~g}, 1.45 \mathrm{mmol})$ was added to a stirred solution of tetramic acid $\mathbf{6 e}$ $(0.40 \mathrm{~g}, 1.45 \mathrm{mmol})$ in dry $\mathrm{CH}_{2} \mathrm{Cl}_{2}(5 \mathrm{~mL})$. The reaction mixture was stirred at room temperature $(16 \mathrm{~h})$ and quenched with saturated aqueous ammonium chloride $(3 \times 5 \mathrm{~mL})$, extracted with methylene chloride $(3 \times 10 \mathrm{~mL})$, dried (anhydrous $\left.\mathrm{Na}_{2} \mathrm{SO}_{4}\right)$, and concentrated to near dryness. The crude product was purified by flash column chromatography [ethyl acetate:hexane (1:2)] to give 7 e $(85 \%)$.

\section{(S)-Methyl 2-( $N$-benzyl-2-bromoacetamido)-3-phenylpropanoate 2a}

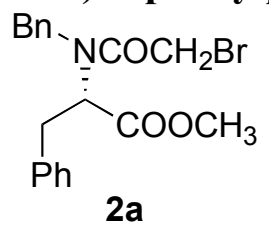

(Procedure A). Methyl (S)- $N$-Benzyl phenylalanine (1.32 g, $5.61 \mathrm{mmol})$ gave 2a (1.93 g, 4.94 mmol, 97\%) as a light yellow oil. $[\alpha]^{22}-142.0$ (c 2.12, $\left.\mathrm{CDCl}_{3}\right) .{ }^{1} \mathrm{H}$ NMR $\left(300 \mathrm{MHz}, \mathrm{CDCl}_{3}\right)$ (mixture of amide rotamers in solution): $\delta 3.25(\mathrm{~m}, 1 \mathrm{H}), 3.38(\mathrm{~m}, 1 \mathrm{H}), 3.66(\mathrm{~s}, 3 \mathrm{H}), 3.78(\mathrm{dd}, J$ $=14.7 \mathrm{~Hz}, J=10.8 \mathrm{~Hz}, 2 \mathrm{H}), 3.83(\mathrm{~d}, J=16.8 \mathrm{~Hz}, 1 \mathrm{H}), 4.25(\mathrm{~m}, 1 \mathrm{H}), 4.51(\mathrm{~d}, J=16.8 \mathrm{~Hz}, 1 \mathrm{H})$, 7.05-7.35 (m, $10 \mathrm{H}) .{ }^{13} \mathrm{C}$ NMR $\left(75 \mathrm{MHz} \mathrm{CDCl}_{3}\right): \delta$ 26.6, 26.8, 35.3, 52.7, 53.7, 62.0, 62.8, 127.2, 127.6, 127.7, 128.0, 128.3, 128.8, 129.0, 129.1, 129.6, 129.7, 129.8, 135.6, 137.9, 167.5, 170.5. IR (thin film): 3030 (w), 2950 (w), 1742 (s), 1652 (s), 1453 (m), 1235 (m), 734 (m), 700 (m) $\mathrm{cm}^{-1}$. Anal. Calcd for $\mathrm{C}_{19} \mathrm{H}_{20} \mathrm{BrNO}_{3}: \mathrm{C}, 58.47 ; \mathrm{H}, 5.18 ; \mathrm{N}, 3.59$. Found: $\mathrm{C}, 58.65 ; \mathrm{H}, 5.22 ; \mathrm{N}$, 3.52 .

\section{(S)-Methyl 2-(N-benzyl-2-bromoacetamido)-4-methyl pentanoate $2 \mathrm{~b}$}

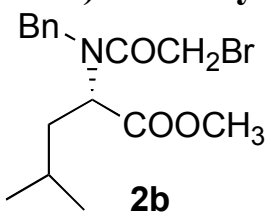

(Procedure A). Methyl $(S)-N$-benzyl leucine (1.32 g, $5.61 \mathrm{mmol})$ gave 2b (1.93 g, $5.41 \mathrm{mmol}$, 97\%) as a light yellow oil. $[\alpha]^{22} \mathrm{D}-26\left(c 1.55, \mathrm{CDCl}_{3}\right) .{ }^{1} \mathrm{H} \mathrm{NMR}\left(300 \mathrm{MHz}, \mathrm{CDCl}_{3}\right)$ (mixture of amide rotamers in solution): $\delta 0.74(\mathrm{~d}, J=6.3 \mathrm{~Hz}, 0.75 \mathrm{H}), 0.81(\mathrm{~d}, J=6.3 \mathrm{~Hz}, 2.25 \mathrm{H}), 0.92(\mathrm{~m}$, $3 \mathrm{H}), 1.55(\mathrm{~m}, 2 \mathrm{H}), 1.85(\mathrm{~m}, 1 \mathrm{H}), 3.55(\mathrm{~s}, 0.75 \mathrm{H}), 3.60(\mathrm{~s}, 2.25 \mathrm{H}), 3.81$ (dd, $J=11.3 \mathrm{~Hz}, J$ $=11.3 \mathrm{~Hz}, 1.5 \mathrm{H}), 3.95,4.15(\mathrm{AX}, J=11.1 \mathrm{~Hz}, 0.5 \mathrm{H}), 4.70(\mathrm{~m}, 3 \mathrm{H}), 7.34(\mathrm{~m}, 5 \mathrm{H}) .{ }^{13} \mathrm{C} \mathrm{NMR}(75$ $\mathrm{MHz}, \mathrm{CDCl}_{3}$ ) (mixture of amide rotamers in solution): $\delta 22.6,23.0,25.3,25.4,27.0,27.3,38.6$, 39.0, 47.4, 51.1, 52.5, 52.8, 57.0, 59.6, 126.95, 127.03, 127.5, 128.0, 128.3, 128.7, 128.7, 129.3, 136.7, 137.9, 168.3, 168.5, 171.3, 171.8. IR (thin film): $2956(\mathrm{~m}), 1742(\mathrm{~s}), 1656(\mathrm{~s}), 1438(\mathrm{~m})$, $1412(\mathrm{~m}), 1244(\mathrm{~m}), 1204(\mathrm{~m}), 1171(\mathrm{~m}), 731(\mathrm{~m}), 699(\mathrm{~m}) \mathrm{cm}^{-1}$. Anal. Calcd for $\mathrm{C}_{16} \mathrm{H}_{22} \mathrm{BrNO}_{3}$ : C, 53.94; H, 6.24; N, 3.93. Found: C, 54.09; H, 6.31; N, 3.97. 
(S)-Methyl 2-(Bromo-N-(4-methoxybenzyl)acetamido)-3-phenylpropanoate 2c

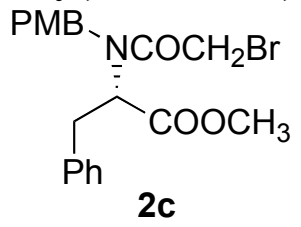

(Procedure A). Methyl (S)-N-4-methoxybenzylphenylalanine (1.5 g, $5.0 \mathrm{mmol})$ gave $2 \mathrm{c}(1.98 \mathrm{~g}$, $4.71 \mathrm{mmol}, 94 \%)$ as a viscous liquid. $[\alpha]^{22} \mathrm{D}-113\left(c \mathrm{2} .2, \mathrm{CHCl}_{3}\right) .{ }^{1} \mathrm{H} \mathrm{NMR}\left(\mathrm{CDCl}_{3}, 300 \mathrm{MHz}\right): \delta$ $3.25(\mathrm{dd}, J=14.05,9.78 \mathrm{~Hz}, 1 \mathrm{H}), 3.38(\mathrm{dd}, J=18.64,9.98 \mathrm{~Hz}, 1 \mathrm{H}), 3.67$ (s, $2.7 \mathrm{H}), 3.73$ (t, $J=$ $4.2 \mathrm{~Hz}, 1.3 \mathrm{H}), 3.78(\mathrm{~s}, 3 \mathrm{H}), 3.85(\mathrm{dd}, J=21.11,10.32 \mathrm{~Hz}, 2 \mathrm{H}), 4.18(\mathrm{~m}, 0.8 \mathrm{H}), 4.62(\mathrm{~d}, J=$ $14.4 \mathrm{~Hz}, 1.2 \mathrm{H}), 6.81,(\mathrm{~d}, J=8.72 \mathrm{~Hz}, 2 \mathrm{H}), 7.03$ (d, $J=8.71 \mathrm{~Hz}, 2 \mathrm{H}), 7.15(\mathrm{~m}, 2 \mathrm{H}), 7.28$ (m, 3 H). ${ }^{13} \mathrm{C} \mathrm{NMR}\left(\mathrm{CDCl}_{3}, 75 \mathrm{MHz}\right): \delta 26.3,34.6,52.1,52.9,55.1,61.2,113.9,126.6,126.7,128.5$, 128.6, 129.3, 137.5, 159.2, 167.0, 170.0. IR (Chloroform): 2951 (w), 2360 (s), 1741 (s), 1653 (s), $1513(\mathrm{~m}), 1456$ (m), 1249 (m), 1178 (m), $824(\mathrm{~s}), 702(\mathrm{~m}) \mathrm{cm}^{-1}$. HRMS (CI+CH 4$)$ Calcd for $\left[\mathrm{C}_{20} \mathrm{H}_{22} \mathrm{BrNO}_{4}+\mathrm{H}^{+}\right]: 420.0811$; Found. $[\mathrm{M}+\mathrm{H}]^{+}: 420.0815$.

\section{(S)-Methyl 2-(2-Bromo-N-(4-methoxybenzyl)acetamido)-4-methylpentanoate 2d}

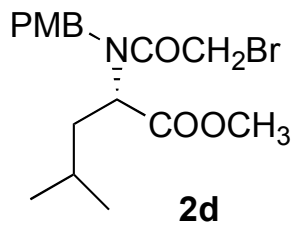

(Procedure A). Methyl (S)-N-4-methoxybenzyl leucine (4.42 g, $16.65 \mathrm{mmol})$ gave 2d (6.22 g, $16.10 \mathrm{mmol}, 97 \%)$ as a viscous liquid. $[\alpha]^{22}{ }_{\mathrm{D}}-52\left(c\right.$ 5.2, $\left.\mathrm{CHCl}_{3}\right) .{ }^{1} \mathrm{H} \mathrm{NMR}\left(\mathrm{CDCl}_{3}, 300 \mathrm{MHz}\right)$ (mixture of amide rotamers in solution): $\delta 0.78(\mathrm{~d}, J=6.69 \mathrm{~Hz}, 0.6 \mathrm{H}), 0.83(\mathrm{~d}, J=6.32 \mathrm{~Hz}, 2.4$ H), 0.89 (d, $J=6.36 \mathrm{~Hz}, 2.7 \mathrm{H}), 0.93(\mathrm{~d}, J=6.28 \mathrm{~Hz}, 0.3 \mathrm{H}), 1.58(\mathrm{~m}, 2 \mathrm{H}), 1.85(\mathrm{~m}, 1 \mathrm{H}), 3.55$ $(\mathrm{s}, 0.7 \mathrm{H}), 3.62(\mathrm{~s}, 2.3 \mathrm{H}), 3.77(\mathrm{~s}, 0.6 \mathrm{H}), 3.80(\mathrm{~s}, 2.4 \mathrm{H}), 3.82(\mathrm{~d}, J=1.27 \mathrm{~Hz}, 1 \mathrm{H}), 4.03(\mathrm{dd}, J$ $=16.56,11.25 \mathrm{~Hz}, 0.4 \mathrm{H}), 4.48(\mathrm{~d}, J=17.23 \mathrm{~Hz}, 0.6 \mathrm{H}), 4.54(\mathrm{~s}, 1 \mathrm{H}), 4.63(\mathrm{~s}, 1 \mathrm{H}), 4.68(\mathrm{~s}, 0.3$ $\mathrm{H}), 4.76(\mathrm{t}, J=6.85 \mathrm{~Hz}, 0.7 \mathrm{H}), 6.81(\mathrm{~d}, J=8.60 \mathrm{~Hz}, 0.5 \mathrm{H}), 6.88(\mathrm{~d}, J=8.88 \mathrm{~Hz}, 1.5 \mathrm{H}), 7.15$ $(\mathrm{d}, J=8.62 \mathrm{~Hz}, 0.4 \mathrm{H}), 7.20(\mathrm{~d}, J=8.73 \mathrm{~Hz}, 1.6 \mathrm{H}) .{ }^{13} \mathrm{C} \mathrm{NMR}\left(\mathrm{CDCl}_{3}, 75 \mathrm{MHz}\right)$ (mixture of amide rotamers in solution): $\delta 22.1,22.6,24.9,26.8,38.1,50.3,52.0,55.3,56.5,114.2,127.9$, 127.9, 129.0, 159.2, 167.7, 171.4. IR (Chloroform): 2956 (w), 2360 (s), 1734 (s), 1717 (s), 1558 (m), $1258(\mathrm{~m}), 821(\mathrm{~m}) \mathrm{cm}^{-1}$. HRMS $\left(\mathrm{CI}+\mathrm{CH}_{4}\right)$ Calcd for $\left[\mathrm{C}_{17} \mathrm{H}_{24} \mathrm{BrNO}_{4}+\mathrm{H}^{+}\right]$: 386.0968; Found. $[\mathrm{M}+\mathrm{H}]^{+}: 386.0963$. 
(2S, 3S)-Methyl 2-(2-bromo- $N$-(4-methoxybenzyl)acetamido)-3-methylpentanoate 2e<smiles>[R16]N(C(=O)OC)[C@@H](C(=O)OC)[C@@H](C)CC</smiles>

(Procedure A). Methyl $(S)-N$-4-methoxybenzyl isoleucine $(1.4 \mathrm{~g}, 3.62 \mathrm{mmol})$ gave $2 \mathrm{e}(1.86 \mathrm{~g}$, $4.81 \mathrm{mmol}, 91 \%)$ as a viscous liquid. $[\alpha]^{22}{ }_{\mathrm{D}}-57$ (c 2.5, $\left.\mathrm{CHCl}_{3}\right) .{ }^{1} \mathrm{H} \mathrm{NMR}\left(\mathrm{CDCl}_{3}, 300 \mathrm{MHz}\right)$ : (mixture of amide rotamers) $\delta 0.73(\mathrm{t}, J=7.28 \mathrm{~Hz}, 0.7 \mathrm{H}), 0.87(\mathrm{t}, J=8.35 \mathrm{~Hz}, 2.3 \mathrm{H}), 0.93(\mathrm{~d}, J$ $=6.58 \mathrm{~Hz}, 3 \mathrm{H}), 1.12(\mathrm{~m}, 1 \mathrm{H}), 1.55(\mathrm{~m}, 1 \mathrm{H}), 2.10(\mathrm{~m}, 1 \mathrm{H}), 3.49(\mathrm{~s}, 3 \mathrm{H}), 3.74(\mathrm{~d}, J=2.96 \mathrm{~Hz}$, $1 \mathrm{H}), 3.77(\mathrm{~s}, 0.5 \mathrm{H}), 3.79(\mathrm{~s}, 2.5 \mathrm{H}), 4.02(\mathrm{t}, J=7.83 \mathrm{~Hz}, 0.5 \mathrm{H}), 4.15(\mathrm{~d}, J=11.54 \mathrm{~Hz}, 0.3 \mathrm{H})$, $4.47(\mathrm{~d}, J=15.24 \mathrm{~Hz}, 0.2 \mathrm{H}), 4.68(\mathrm{~s}, 1.8 \mathrm{H}), 4.75(\mathrm{~s}, 0.2 \mathrm{H}), 4.95(\mathrm{~d}, J=10.48 \mathrm{~Hz}, 1 \mathrm{H}), 6.79$ $(\mathrm{d}, J=8.58 \mathrm{~Hz}, 0.5 \mathrm{H}), 6.87(\mathrm{~d}, J=8.58 \mathrm{~Hz}, 1.5 \mathrm{H}), 7.06(\mathrm{~d}, J=8.62 \mathrm{~Hz}, 1.5 \mathrm{H}), 7.18(\mathrm{~d}, J=$ $8.51 \mathrm{~Hz}, 0.5 \mathrm{H}) .{ }^{13} \mathrm{C} \mathrm{NMR}\left(\mathrm{CDCl}_{3}, 75 \mathrm{MHz}\right): \delta 10.7,11.0,15.8,24.3,26.7,34.1,34.2,48.1$, 51.7, 55.2, 60.9, 66.0, 113.5, 114.2, 126.7, 128.3, 129.3, 158.9, 168.2, 170.5. IR (Chloroform): 2964 (w), 2360 (m), 1740 (s), 1655 (s), 1457 (m), 1336 (m), 1249 (m), 1139 (m), 817(m). cm ${ }^{-1}$. HRMS $\left(\mathrm{CI}+\mathrm{CH}_{4}\right)$ Calcd for $\left[\mathrm{C}_{17} \mathrm{H}_{24} \mathrm{BrNO}_{4}+\mathrm{H}^{+}\right]$: 386.0961 ; Found. $[\mathrm{M}+\mathrm{H}]^{+}: 386.0961$.

\section{(2S,3S)-Methyl 2-(2-Bromo- $N$-(3,4-dimethoxybenzyl) acetamido)-3-methylpentanoate $2 f$}

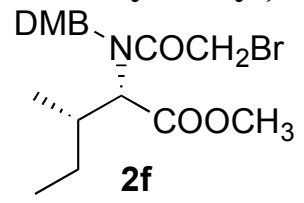

(Procedure A). Methyl (S)-N-3, 4-dimethoxybenzyl isoleucine (0.83 g, $2.79 \mathrm{mmol})$ gave $2 \mathbf{f}(1.11$ g, $2.66 \mathrm{mmol}, 96 \%)$ as a light yellow oil. $[\alpha]^{22}{ }_{\mathrm{D}}-47\left(c 4.92, \mathrm{CHCl}_{3}\right) .{ }^{1} \mathrm{H} \mathrm{NMR}(300 \mathrm{MHz}$, $\left.\mathrm{CDCl}_{3}\right)$ : (mixture of amide rotamers in solution): $\delta 0.71(\mathrm{t}, J=7.2 \mathrm{~Hz}, 0.7 \mathrm{H}), 0.89(\mathrm{t}, J=7.42$ $\mathrm{Hz}, 2.3 \mathrm{H}), 0.94(\mathrm{~d}, J=6.6 \mathrm{~Hz}, 3 \mathrm{H}), 1.18(\mathrm{~m}, 1 \mathrm{H}), 1.52(\mathrm{~m}, 1 \mathrm{H}), 2.08(\mathrm{~m}, 1 \mathrm{H}), 3.52(\mathrm{~s}, 3 \mathrm{H})$, $3.75(\mathrm{dd}, J=13.5 \mathrm{~Hz}, 10.5 \mathrm{~Hz}, 1.3 \mathrm{H}), 3.87$ (s, $6 \mathrm{H}), 4.12(\mathrm{~m}, 0.7 \mathrm{H}), 4.53$ (d, $J=15.3 \mathrm{~Hz}, 0.3$ H), $4.69(\mathrm{dd}, J=24.6,17.4 \mathrm{~Hz}, 2 \mathrm{H}), 4.94(\mathrm{~d}, J=10.2 \mathrm{~Hz}, 0.7 \mathrm{H}), 6.65-6.83(\mathrm{~m}, 3 \mathrm{H}) .{ }^{13} \mathrm{C}$ NMR $\left(300 \mathrm{MHz}, \mathrm{CDCl}_{3}\right)$ (mixture of amide rotamers in solution): 10.4, 10.7, 15.5, 15.6, 24.1, 24.9, 26.2, 26.4, 30.4, 33.8, 33.9, 45.8, 48.2, 51.3, 51.6, 55.5, 60.8, 65.7, 108.6, 110.3, 110.9, 117.1, 119.9, 128.6, 129.6, 147.6, 147.9, 148.3, 148.9, 167.8, 169.6, 170.0. IR $\left(\mathrm{CDCl}_{3}\right): 2964(\mathrm{w}), 2361$ (s), 1740 (s), 1655 (s), 1517 (m), 1463 (m), 1311 (m), 1261 (m), 1027 (m), 915 (m), 806 (m), $732(\mathrm{~m}) \mathrm{cm}^{-1}$. HRMS (CI+CH$)$ Calcd for $\mathrm{C}_{18} \mathrm{H}_{26} \mathrm{BrNO}_{5}$ : 415.0994; Found. [M] ${ }^{+}: 415.0980$. 


\section{(2S, 3S)-Methyl 2-(N-benzyl-2-bromoacetamido)-3-methylpentanoate 2g<smiles>CC[C@H](C)[C@H](C(=O)OC)N(Cc1ccccc1)C(=O)O</smiles>

(Procedure A). Methyl $(S)-N$-benzyl isoleucine $(3.60 \mathrm{~g}, 15.3 \mathrm{mmol})$ gave 2g (5.45 g, $15.3 \mathrm{mmol}$, $100 \%)$ as a light brown oil. $[\alpha]^{19}{ }_{\mathrm{D}}-52\left(c\right.$ 1.2, $\left.\mathrm{CHCl}_{3}\right){ }^{1} \mathrm{H} \mathrm{NMR}\left(300 \mathrm{MHz}, \mathrm{CDCl}_{3}\right)$ (mixture of amide rotamers in solution): $\delta 0.69(\mathrm{t}, J=7.8 \mathrm{~Hz}, 0.5 \mathrm{H}), 0.88(\mathrm{t}, J=7.49 \mathrm{~Hz}, 2.5 \mathrm{H}), 0.93(\mathrm{~d}, J$ $=6.58 \mathrm{~Hz}, 3 \mathrm{H}), 1.14(\mathrm{~m}, 1 \mathrm{H}), 1.52(\mathrm{~m}, 1 \mathrm{H}), 2.10(\mathrm{~m}, 1 \mathrm{H}), 3.46(\mathrm{~s}, 3 \mathrm{H}), 3.73(\mathrm{~s}, 1.5 \mathrm{H}), 4.06$ $(\mathrm{m}, 0.5 \mathrm{H}), 4.17(\mathrm{~m}, 0.3 \mathrm{H}), 4.55(\mathrm{~d}, J=15.0 \mathrm{~Hz}, 0.3 \mathrm{H}), 4.75(\mathrm{~s}, 1.7 \mathrm{H}), 5.02(\mathrm{~d}, J=10.5 \mathrm{~Hz}, 0.7$ $\mathrm{H}), 7.11-7.38(\mathrm{~m}, 5 \mathrm{H}) .{ }^{13} \mathrm{C} \mathrm{NMR}\left(300 \mathrm{MHz}, \mathrm{CDCl}_{3}\right)$ (mixture of amide rotamers in solution): 10.7, 15.7, 24.3, 26.5, 34.2, 48.3, 51.6, 60.7, 125.3, 127.5, 128.2, 128.9, 130.3, 136.5, 168.4, 170.5. IR ( $\left.\mathrm{CDCl}_{3}\right): 2966$ (w), 2360 (s), 1741(s), 1717(s), 1412 (m), 1362 (m), 1199 (m), 735 (m) $\mathrm{cm}^{-1}$. HRMS $\left(\mathrm{CI}+\mathrm{CH}_{4}\right)$ Calcd for $\left[\mathrm{C}_{16} \mathrm{H}_{22} \mathrm{BrNO}_{3}+\mathrm{H}^{+}\right]: 356.0861$; Found. $[\mathrm{M}+\mathrm{H}]^{+}: 356.0847$.

\section{1-(2-Bromoacetyl)- $N$-methoxy- $N$-methylpyrrolidine-2-carboxamide $2 \mathrm{~h}$}

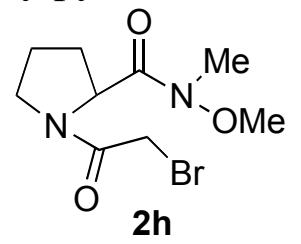

$N$-Methylmorpholine $(0.46 \mathrm{~g}, 4.57 \mathrm{mmol})$ was added to a solution of $N$-Boc racemic proline (0.19 g, $0.915 \mathrm{mmol})$ in $\mathrm{CH}_{2} \mathrm{Cl}_{2}(5 \mathrm{~mL})$ at $-15{ }^{\circ} \mathrm{C}$ followed by dropwise addition of isobutylchloroformate $(0.13 \mathrm{~g}, 1.37 \mathrm{mmol})$. The resulting solution was stirred at this temperature for $15 \mathrm{~min} . \mathrm{N}, O$-Dimethylhydroxylamine hydrochloride $(0.26 \mathrm{~g}, 2.74 \mathrm{mmol})$ was added in one portion and the mixture stirred for $1 \mathrm{~h}$ at $-15^{\circ} \mathrm{C}$, and $3 \mathrm{~h}$ at room temperature. Water was added to the reaction mixture, the aqueous layer extracted with $\mathrm{CH}_{2} \mathrm{Cl}_{2}(3 \times 5 \mathrm{~mL})$, and the combined organic extracts were dried $\left(\mathrm{Na}_{2} \mathrm{SO}_{4}\right)$. The solvent was removed under reduced pressure and the crude reaction mixture was dissolved in methylene chloride $(5 \mathrm{~mL})$. Trifluoroacetic acid $(4.82 \mathrm{~g}$, $42.3 \mathrm{mmol}$ ) was added, and the mixture was stirred for $22 \mathrm{~h}$. Procedure A was followed to give 2h (0.76 g, $2.72 \mathrm{mmol}, 71 \%)$ as a yellow oil. ${ }^{1} \mathrm{H}$ NMR $\left(300 \mathrm{MHz}, \mathrm{CDCl}_{3}\right)($ mixture of amide rotamers in solution): $\delta 1.98(\mathrm{~m}, 2 \mathrm{H}), 2.2(\mathrm{~m}, 2 \mathrm{H}), 3.21(\mathrm{~s}, 2.4 \mathrm{H}), 3.23(\mathrm{~s}, 0.6 \mathrm{H}), 3.71(\mathrm{~m}, 2$ H), $3.81(\mathrm{~s}, 0.6 \mathrm{H}), 3.82(\mathrm{~d}, J=10.8 \mathrm{~Hz}, 1 \mathrm{H}), 3.83(\mathrm{~s}, 2.4 \mathrm{H}), 3.92(\mathrm{~d}, J=10.8 \mathrm{~Hz}, 1 \mathrm{H}), 4.92(\mathrm{~m}$, $1 \mathrm{H}) .{ }^{13} \mathrm{C} \mathrm{NMR}\left(75 \mathrm{MHz}, \mathrm{CDCl}_{3}\right.$ ) (mixture of amide rotamers in solution): $\delta 22.3,24.7,27.3$, 28.1, 28.5, 28.9, 31.9, 45.0, 47.5, 47.7, 56.9, 57.9, 61.2, 61.6, 69.2, 164.9, 165.1, 171.6. IR (thin film): $2974(\mathrm{~m}), 2878(\mathrm{~m}), 1651$ (s), 1446 (s), $1179(\mathrm{~m}), 993(\mathrm{~m}) \mathrm{cm}^{-1}$. Anal. Calcd for $\mathrm{C}_{9} \mathrm{H}_{15} \mathrm{BrN}_{2} \mathrm{O}_{3}: \mathrm{C}, 38.73 ; \mathrm{H}, 5.42 ; \mathrm{N}, 10.04$. Found: C, 38.74; H, 5.47; N, 10.08. 
(S)-(N-Benzyl- $N$-(2-bromopropionyl))phenylalanine Methyl Esters 2i

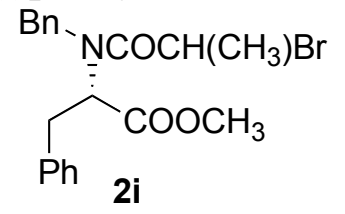

(Procedure A). Methyl (S)- $N$-benzylphenylalanine $(0.64 \mathrm{~g}, 2.38 \mathrm{mmol})$ gave $2 \mathbf{i}(0.93 \mathrm{~g}, 2.3$ mmol, 97\%) as a light yellow oil. $[\alpha]^{22}-126$ (c 1.78, $\left.\mathrm{CDCl}_{3}\right) .{ }^{1} \mathrm{H}$ NMR $\left(300 \mathrm{MHz}, \mathrm{CDCl}_{3}\right)$ (mixture of amide rotamers in solution): $\delta 1.34(\mathrm{~d}, J=6.3 \mathrm{~Hz}, 0.48 \mathrm{H}), 1.79(\mathrm{~d}, J=6.6 \mathrm{~Hz}, 2.52$ H), 3.18 (m, $1 \mathrm{H}), 3.26$ (s, $0.48 \mathrm{H}), 3.36(\mathrm{dd}, J=14.0 \mathrm{~Hz}, J=6.5 \mathrm{~Hz}, 1 \mathrm{H}), 3.54$ (s, $2.52 \mathrm{H}), 4.19$, 4.53 (AX, $J=15.9 \mathrm{~Hz}, 1.84 \mathrm{H}), 4.40$ (q, $J=6.6 \mathrm{~Hz}, 1 \mathrm{H}), 4.55$ (m, $1 \mathrm{H}), 4.89$ (m, $0.16 \mathrm{H}), 7.04-$ $7.35(\mathrm{~m}, 10 \mathrm{H}) .{ }^{13} \mathrm{C} \mathrm{NMR}\left(75 \mathrm{MHz}, \mathrm{CDCl}_{3}\right)$ (mixture of amide rotamers in solution): $\delta 21.7$, 22.1, 35.6, 36.3 , 38.0, 39.0, 52.1, 52.5, 52.6, 60.7, 62.0, 127.2, 127.5, 127.6, 127.8, 128.1, 128.3, 128.6, 129.0, 129.2, 129.5, 129.7, 136.0, 137.8, 170.4, 170.7. IR (thin film): 3064 (w), 3029 (m), 2950 (m), 1745 (s), 1660 (s), 1496 (m), 1454 (s), 1350 (m), 1228 (s), 1168 (m), 1064 (w), 912 (w), 734 (s), 700 (s) $\mathrm{cm}^{-1}$. Anal. Calcd for $\mathrm{C}_{20} \mathrm{H}_{22} \mathrm{BrNO}_{3}: \mathrm{C}, 59.41 ; \mathrm{H}, 5.50 ; \mathrm{N}, 3.46$. Found: C, 59.30; H, 5.45; N, 3.45 .

\section{(2S)-Methyl 2-( $N$-benzyl-2-bromopropanamido)-4-methyl pentanoate $2 \mathrm{j}$}

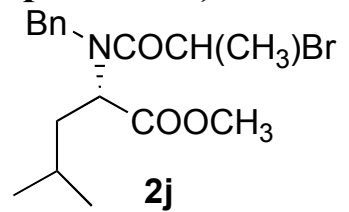

(Procedure A). Methyl (S)- $N$-benzyl leucine $(0.22 \mathrm{~g}, 1.71 \mathrm{mmol})$ gave $2 \mathbf{j}(0.278 \mathrm{~g}, 0.75 \mathrm{mmol}$, 97\%) as a colorless oil. $[\alpha]^{24}-57\left(c\right.$ 1.52, $\left.\mathrm{CDCl}_{3}\right) .{ }^{1} \mathrm{H}$ NMR $\left(300 \mathrm{MHz}, \mathrm{CDCl}_{3}\right)$ (mixture of amide rotamers in solution): $\delta 0.75(\mathrm{~m}, 0.6 \mathrm{H}), 0.90(\mathrm{~m}, 5.4 \mathrm{H}), 1.63(\mathrm{~m}, 2 \mathrm{H}), 1.76(\mathrm{~d}, J=6.6$ $\mathrm{Hz}, 3 \mathrm{H}), 1.86(\mathrm{~m}, 1 \mathrm{H}), 3.47(\mathrm{~s}, 2.7 \mathrm{H}), 3.54$ (s, $0.3 \mathrm{H}), 4.42$ (q, J=6.6 Hz, $1 \mathrm{H}), 4.72$ (m, 2.1 $\mathrm{H}), 5.13(\mathrm{~m}, 0.9 \mathrm{H}), 7.18-7.35(\mathrm{~m}, 5 \mathrm{H}) .{ }^{13} \mathrm{C} \mathrm{NMR}\left(75 \mathrm{MHz}, \mathrm{CDCl}_{3}\right)$ (mixture of amide rotamers in solution): $\delta 21.8,22.7,22.9,23.2,25.2,38.9,39.5,49.3,51.2,52.3,52.6,55.8,58.0,126.5$, 128.1, 128.7, 129.3, 129.4, 136.5, 136.9, 171.0, 171.8. IR (thin film): 2956 (m), $2870(\mathrm{w}), 1742$ (s), 1662 (s), 1451 (m), 1234 (m), 1204 (m), 1168 (m), 1132 (w), 733 (m), 698 (m) cm ${ }^{-1}$. Anal. Calcd for $\mathrm{C}_{17} \mathrm{H}_{24} \mathrm{BrNO}_{3}$ : C, 55.14; H, 6.55; N, 3.78. Found: C, 55.20; H, 6.59; N, 3.41.

\section{(S)-Methyl 2-(N-benzyl-2-bromo-2-methylpropanamido)-4-methylpentanoate 2k}

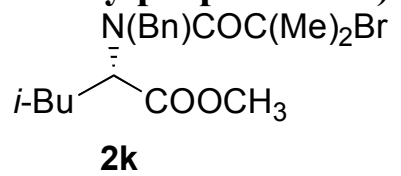

(Procedure A). Leucine methyl ester $(0.77 \mathrm{~g}, 3.28 \mathrm{mmol})$ gave the dimethyl bromoacyl derivative $2 \mathbf{k}(1.25 \mathrm{~g}, 3.25 \mathrm{mmol}, 99 \%)$ as a colorless oil. $[\alpha]^{23} \mathrm{D}-67\left(c 1.36, \mathrm{CDCl}_{3}\right) .{ }^{1} \mathrm{H}$ NMR $\left(300 \mathrm{MHz}, \mathrm{CDCl}_{3}\right): \delta 0.65$ (br s, $\left.3 \mathrm{H}\right), 0.83$ (br d, $\left.J=6.3 \mathrm{~Hz}, 3 \mathrm{H}\right), 1.40(\mathrm{~m}, 1 \mathrm{H}), 1.59(\mathrm{~m}, 1 \mathrm{H})$, 2.00 (br s, 3 H), 2.03 (s, 3 H), 2.22 (br s, 1 H), 3.54 (br s, 1 H), 3.66 (s, 3 H), 5.07 (m, 2 H), 7.25- 
$7.50(\mathrm{~m}, 5 \mathrm{H}) .{ }^{13} \mathrm{C} \mathrm{NMR}\left(75 \mathrm{MHz}, \mathrm{CDCl}_{3}\right): \delta 22.3,23.3,25.9,32.4,33.7,39.2,52.4,55.1,57.3$, 59.8, 128.5, 128.7, 129.0, 136.2, 171.0, 171.8. IR (thin film): 2955 (s), 2869 (m), 1745 (s), 1643 (s), 1464 (s), 1433 (s), 1240 (s), 1203 (s), 1168 (s), 1110 (m), 999 (w), 731 (m), 699 (m) cm ${ }^{-1}$. Anal. Calcd for $\mathrm{C}_{18} \mathrm{H}_{26} \mathrm{BrNO}_{3}$ : C, 56.25; H, 6.83; N, 3.64. Found: C, 56.33; H, 6.91; N, 3.64 .

\section{(S)-Methyl 2-( $N$-benzyldeuteroacetamido)-4-methylpentanoate $4 \mathrm{~b}$}

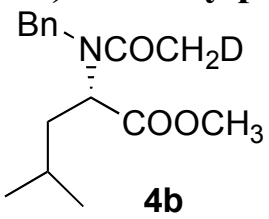

Lithium telluride $(0.15 \mathrm{~mL}, 0.12 \mathrm{mmol}, 0.25 \mathrm{M}$ suspension) was added to a solution of haloacylamino acid ester $\mathbf{2 b}(0.22 \mathrm{~g}, 0.63 \mathrm{mmol})$ in $\mathrm{CD}_{3} \mathrm{OD}$ :THF $(9: 1)$, stirred at room temperature for $20 \mathrm{~min}$., filtered through a celite pad, washed with $\mathrm{CD}_{3} \mathrm{OD}$, and concentrated to dryness to give a liquid. The crude ${ }^{1} \mathrm{H}$ NMR spectrum indicated deuterated product $\mathbf{4 b}:{ }^{1} \mathrm{H}$ NMR $\left(\mathrm{CDCl}_{3}, 300 \mathrm{MHz}\right): \delta 0.75(\mathrm{~d}, J=6.3 \mathrm{~Hz}, 3 \mathrm{H}), 0.85(\mathrm{~d}, J=6.3 \mathrm{~Hz}, 3 \mathrm{H}), 1.58-1.62(\mathrm{~m}, 2 \mathrm{H})$, 2.09-2.11 (m, $\left.2 \mathrm{H}, \mathrm{NCOCH}_{2} \mathrm{D}\right), 2.24-2.25(\mathrm{~m}, 1 \mathrm{H}), 3.51(\mathrm{~s}, 3 \mathrm{H}), 3.70(\mathrm{~d}, J=8.6 \mathrm{~Hz}, 1 \mathrm{H}), 3.95$ $(\mathrm{d}, J=12.2 \mathrm{~Hz}, 1 \mathrm{H}), 4.48(\mathrm{~d}, J=8.6 \mathrm{~Hz}, 1 \mathrm{H}), 7.17-7.33(\mathrm{~m}, 5 \mathrm{H})$.

\section{(S)-5-i-Butyl-1-(4-Methoxybenzyl)pyrrolidine-2, 4-dione 6d}

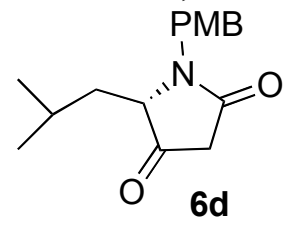

(Procedure B). Compound 2d (0.45 g, $1.29 \mathrm{mmol})$ gave crude 6d (0.26 g, $0.94 \mathrm{mmol}, 72 \%)$ as an unstable colorless liquid. ${ }^{1} \mathrm{H} \mathrm{NMR}\left(\mathrm{CDCl}_{3}, 300 \mathrm{MHz}\right): \delta 0.81(\mathrm{~d}, J=6.8 \mathrm{~Hz}, 3 \mathrm{H}), 0.85(\mathrm{~d}, J=$ $6.8 \mathrm{~Hz}, 3 \mathrm{H}), 1.54-1.61(\mathrm{~m}, 2 \mathrm{H}), 1.72(\mathrm{~m}, 1 \mathrm{H}), 2.82(\mathrm{~s}, 2 \mathrm{H}), 3.73(\mathrm{~s}, 3 \mathrm{H}), 3.72(\mathrm{~d}, J=12.2 \mathrm{~Hz}$, $1 \mathrm{H}), 3.95(\mathrm{~d}, J=4.2 \mathrm{~Hz}, 1 \mathrm{H}), 5.20(\mathrm{~d}, J=12.2 \mathrm{~Hz}, 1 \mathrm{H}), 6.72(\mathrm{~d}, J=8.50 \mathrm{~Hz}, 2 \mathrm{H}), 7.23(\mathrm{~d}, J=$ $8.50 \mathrm{~Hz}, 2 \mathrm{H})$.

(S)-5-sec-Butyl-1-(4-Methoxybenzyl)pyrrolidine-2, 4-dione 6e

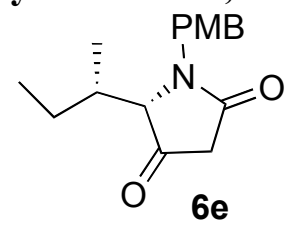

(Procedure B). Compound 2e (0.45 g, $1.16 \mathrm{mmol})$ gave 6e $(0.21 \mathrm{~g}, 0.76 \mathrm{mmol}, 65 \%)$ as an unstable colorless liquid. $[\alpha]^{21} \mathrm{D}-97\left(c\right.$ 2.5, $\left.\mathrm{CHCl}_{3}\right) .{ }^{1} \mathrm{H} \mathrm{NMR}\left(\mathrm{CDCl}_{3}, 300 \mathrm{MHz}\right)$ : 0.82-0.89 (m, 6 H), $1.46(\mathrm{~m}, 2 \mathrm{H}), 1.89(\mathrm{~m}, 1 \mathrm{H}), 2.99(\mathrm{~s}, 2 \mathrm{H}), 3.68(\mathrm{~d}, J=3.02 \mathrm{~Hz}, 1 \mathrm{H}), 3.80(\mathrm{~s}, 3 \mathrm{H}), 3.89$ (d, $J=14.64 \mathrm{~Hz}, 1 \mathrm{H}), 5.28(\mathrm{~d}, J=14.77 \mathrm{~Hz}, 1 \mathrm{H}), 6.87$ (d, $J=8.63 \mathrm{~Hz}, 2 \mathrm{H}), 7.19$ (d, $J=8.60 \mathrm{~Hz}$, $2 \mathrm{H}) .{ }^{13} \mathrm{C} \mathrm{NMR}\left(\mathrm{CDCl}_{3}, 75 \mathrm{MHz}\right): 12.0,13.5,25.1,35.1,42.7,42.9,55.2,68.4,114.2,127.1$, 
129.7, 159.3, 169.0, 206.5. IR (chloroform): 2962 (w), 2339 (m), 1769 (s), 1697 (s), 1513 (m), $1457(\mathrm{~m}), 1247(\mathrm{~m})$.

\section{Tetrahydro-1H-pyrrolizine-1,3(2H)-dione $6 h^{33}$}

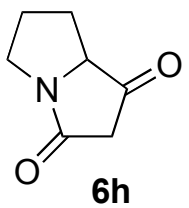

Lithium telluride $(0.24 \mathrm{~mL}, 0.06 \mathrm{mmol}, 0.25 \mathrm{M}$ suspension) was added to a solution of $\mathbf{2 h}$ (14 $\mathrm{mg}, 0.050 \mathrm{mmol})$ in THF $(0.3 \mathrm{~mL})$ followed by a THF solution of LHMDS $(0.05 \mathrm{~mL}, 0.05$ mmol, $1 \mathrm{M})$ at room temperature. The mixture was stirred for $2 \mathrm{~h}$, poured into $3 \%$ aq. $\mathrm{HCl}(5$ $\mathrm{mL})$, and extracted with methylene chloride $(3 \times 2 \mathrm{~mL})$. The organic layer was dried $\left(\mathrm{Na}_{2} \mathrm{SO}_{4}\right)$ and chromatographed on silica gel $\left(\mathrm{CHCl}_{3} / \mathrm{EtOH} 30: 1\right)$ to give $\mathbf{6 h}(5.9 \mathrm{mg}, 0.04 \mathrm{mmol}, 80 \%)$, as an unstable solid. ${ }^{1} \mathrm{H}$ NMR $\left(300 \mathrm{MHz}, \mathrm{CDCl}_{3}\right)$ : $\delta$ 1.62-1.70 (m, $\left.2 \mathrm{H}\right), 2.08-2.16(\mathrm{~m}, 2 \mathrm{H}), 3.03$ (dd, $J=21.3 \mathrm{~Hz}, J=1.4 \mathrm{~Hz}, 1 \mathrm{H}), 3.19-3.33(\mathrm{~m}, 1 \mathrm{H}), 3.36-3.42(\mathrm{~m}, 1 \mathrm{H}), 3.95$ (dt, $J=11.7 \mathrm{~Hz}, J=7.5$ $\mathrm{Hz}, 1 \mathrm{H}), 4.18-4.26(\mathrm{~m}, 1 \mathrm{H}) ;{ }^{13} \mathrm{C} \mathrm{NMR}\left(75 \mathrm{MHz}, \mathrm{CDCl}_{3}\right): \delta 26.3,27.1,43.8,45.1,70.5,170.8$, 206.4. IR ( $\left.\mathrm{CDCl}_{3}\right)$ : 2981 (m), 2899 (m), 1768 (s), 1695 (s), 1404 (m), 1375 (m), 1258 (m) $\mathrm{cm}^{-1}$.

\section{(S)-1-Benzyl-5-isobutyl-3,3-dimethylpyrrolidin-2,4-dione 6k}

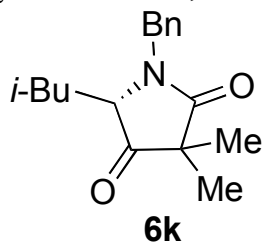

Lithium telluride $(2.5 \mathrm{~mL}, 0.61 \mathrm{mmol}, 0.25 \mathrm{M}$ suspension) was added to a solution of $\mathbf{2 k}(0.23 \mathrm{~g}$, $0.60 \mathrm{mmol})$ in THF $(8.1 \mathrm{~mL})$ at room temperature. The mixture was stirred $(18 \mathrm{~h})$, poured into aqueous $\mathrm{NH}_{4} \mathrm{Cl}(5 \mathrm{~mL})$, and extracted with EtOAc $(3 \times 30 \mathrm{~mL})$. The organic layer was dried $\left(\mathrm{Na}_{2} \mathrm{SO}_{4}\right)$ and chromatographed on silica gel (hexane/EtOAc 3:1) to give 6k (0.13 g, $0.47 \mathrm{mmol}$, $79 \%)$ as a colorless liquid. $[\alpha]^{24}-4\left(c 0.36, \mathrm{CHCl}_{3}\right) .{ }^{1} \mathrm{H} \mathrm{NMR}\left(300 \mathrm{MHz}, \mathrm{CDCl}_{3}\right): \delta 0.79(\mathrm{~d}$, $J=6.3 \mathrm{~Hz}, 3 \mathrm{H}), 0.88(\mathrm{~d}, J=6.6 \mathrm{~Hz}, 3 \mathrm{H}), 1.25(\mathrm{~s}, 3 \mathrm{H}), 1.29(\mathrm{~s}, 3 \mathrm{H}), 1.56(\mathrm{~m}, 2 \mathrm{H}), 1.82(\mathrm{~m}, 1 \mathrm{H})$, $3.69(\mathrm{dd}, J=7.8 \mathrm{~Hz}, J=4.2 \mathrm{~Hz}, 1 \mathrm{H}), 3.97,5.37(\mathrm{AX}, J=14.9 \mathrm{~Hz}, 2 \mathrm{H}), 7.30(\mathrm{~m}, 5 \mathrm{H}) .{ }^{13} \mathrm{C}$ NMR $\left(75 \mathrm{MHz}, \mathrm{CDCl}_{3}\right): \delta 21.4,21.8,22.8,23.6,25.0,38.9,43.9,46.9,61.6,128.4,128.5$, 129.3, 135.9, 176.2, 214.1. IR (thin film): 2960 (m), 2932 (m), 2871 (w), 1766 (s), 1694 (s), 1465 (m), 1416 (s), 1297 (m), 701 (m) cm ${ }^{-1}$. Anal. Calcd for $\mathrm{C}_{17} \mathrm{H}_{23} \mathrm{NO}_{2}: \mathrm{C}, 74.68 ; \mathrm{H}, 8.50 ; \mathrm{N}$, 5.12. Found: C, 74.69; H, 8.54; N, 5.09. 


\section{(S)-1,5-Dibenzyl-4-(tert-butyldiphenylsilyloxy)-1H-pyrrol-2(5H)-one 7a}

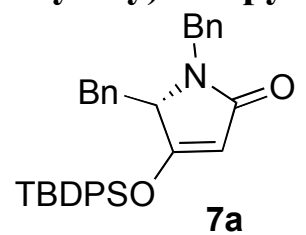

(Procedure C). Compound 2a $(0.50 \mathrm{~g}, 1.27 \mathrm{mmol})$ gave $7 \mathbf{a}(0.36 \mathrm{~g}, 0.69 \mathrm{mmol}, 55 \%)$ as a colorless oil. $[\alpha]^{24}{ }_{\mathrm{D}}-6\left(c \mathrm{1} 1.46, \mathrm{CDCl}_{3}\right) .{ }^{1} \mathrm{H}$ NMR (300 MHz, $\left.\mathrm{CDCl}_{3}\right): \delta 1.09$ (s, $\left.9 \mathrm{H}\right), 3.00$ (dd, $J$ $=14.4 \mathrm{~Hz}, J=6.0 \mathrm{~Hz}, 1 \mathrm{H}), 3.31(\mathrm{dd}, J=14.4 \mathrm{~Hz}, J=4.2 \mathrm{~Hz}, 1 \mathrm{H}), 3.85(\mathrm{~d}, J=15.5 \mathrm{~Hz}, 1 \mathrm{H})$, $4.11(\mathrm{~m}, 1 \mathrm{H}), 4.63(\mathrm{~s}, 1 \mathrm{H}), 5.22(\mathrm{~d}, J=15.5 \mathrm{~Hz}, 1 \mathrm{H}), 6.96-7.61(\mathrm{~m}, 20 \mathrm{H}) .{ }^{13} \mathrm{C} \mathrm{NMR}(75 \mathrm{MHz}$, $\left.\mathrm{CDCl}_{3}\right): \delta 19.8,27.0,36.1,44.1,61.7,102.4,127.4,127.8,128.3,128.5,129.0,129.1,129.8$, $130.7,130.9,131.0,131.1,135.8,136.6,138.0,171.5,172.8$. IR (thin film): 2931 (m), 1682 (s), $1620(\mathrm{~s}), 1430(\mathrm{w}), 1336(\mathrm{w}), 1220(\mathrm{w}), 1114(\mathrm{w}), 738(\mathrm{w}), 700(\mathrm{~s}) \mathrm{cm}^{-1}$. Anal. Calcd for $\mathrm{C}_{34} \mathrm{H}_{35} \mathrm{NO}_{2} \mathrm{Si}$ : C, 78.86; H, 6.83; N, 2.71. Found: C, 78.67; H, 6.79; N, 2.62.

\section{(S)-1-Benzyl-4-(tert-butyldiphenylsilyloxy)-5-isobutyl-1 $H$-pyrrol-2(5H)-one 7 b}

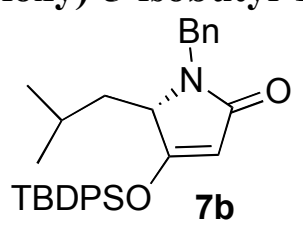

(Procedure C). 2b $(0.47 \mathrm{~g}, 1.32 \mathrm{mmol})$ gave $7 \mathbf{b}(0.52 \mathrm{~g}, 1.07 \mathrm{mmol} 82 \%)$ as a colorless oil. $[\alpha]^{22}{ }_{\mathrm{D}}$ 1.5 (c 1.9, $\left.\mathrm{CDCl}_{3}\right) .{ }^{1} \mathrm{H} \mathrm{NMR}\left(300 \mathrm{MHz}, \mathrm{CDCl}_{3}\right): \delta 0.89$ (d, J=6.6 Hz, $\left.6 \mathrm{H}\right), 1.07$ (s, $\left.9 \mathrm{H}\right), 1.68$ (m, $2 \mathrm{H}), 1.85(\mathrm{~m}, 1 \mathrm{H}), 3.83$ (t, J=5.3 Hz, $1 \mathrm{H}), 3.97$ (d, J=15.5 Hz, $1 \mathrm{H}), 4.65$ (s, $1 \mathrm{H}), 5.13$ (d, $J=15.5 \mathrm{~Hz}, 1 \mathrm{H}), 7.04-7.48$ (m, $15 \mathrm{H}) .{ }^{13} \mathrm{C} \mathrm{NMR}\left(75 \mathrm{MHz}, \mathrm{CDCl}_{3}\right): \delta 19.2,23.1,23.3,25.5,26.3$, $38.3,43.2,59.5,100.9,127.2,127.6,127.9,127.9,128.5,130.3,130.5,131.5,135.2,135.3$, 137.7, 172.0, 172.4. IR (thin film): 2957 (s), 2932 (s), 1686 (s), 1619 (s), 1429 (m), 1405 (m),

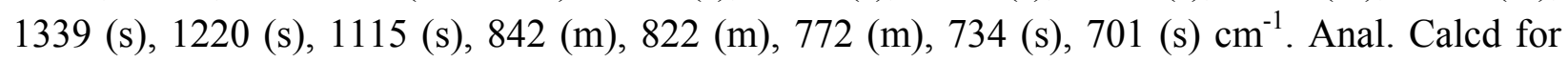
$\mathrm{C}_{31} \mathrm{H}_{37} \mathrm{NO}_{2} \mathrm{Si}$ : C, 76.96; H, 7.72; N, 2.90. Found: C, 77.03; H, 7.77; N, 2.99.

(S)-2-sec-Butyl-1-(4-methoxybenzyl)-5-oxo-2,5-dihydro-1H-pyrrol-3-yl 4-methylbenzenesulfonate 7e

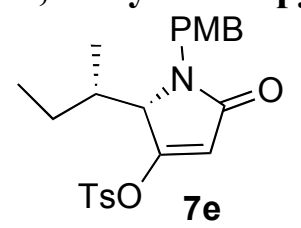

(Procedure E). Isoleucine crude tetramic acid 6e $(0.25 \mathrm{~g}, 0.91 \mathrm{mmol})$ gave $7 \mathrm{e}(0.33 \mathrm{~g}, 0.76$ mmol, 85\%). $[\alpha]^{23}$ D $-40\left(c 2.0, \mathrm{CHCl}_{3}\right) .{ }^{1} \mathrm{H}$ NMR (300 MHz, $\left.\mathrm{CDCl}_{3}\right): \delta 0.58(\mathrm{~d}, J=6.91 \mathrm{~Hz}, 3$ $\mathrm{H}), 0.81(\mathrm{t}, J=7.32 \mathrm{~Hz}, 3 \mathrm{H}), 1.29(\mathrm{~m}, 2 \mathrm{H}), 1.81(\mathrm{~m}, 1 \mathrm{H}), 2.46(\mathrm{~s}, 3 \mathrm{H}), 3.73(\mathrm{~d}, J=2.68 \mathrm{~Hz}, 1$ H), $3.78(\mathrm{~s}, 3 \mathrm{H}), 3.82(\mathrm{~d}, J=14.87 \mathrm{~Hz}, 1 \mathrm{H}), 5.06(\mathrm{~d}, J=15.06 \mathrm{~Hz}, 1 \mathrm{H}), 5.78(\mathrm{~d}, J=3.20 \mathrm{~Hz}, 1$ $\mathrm{H}), 6.81(\mathrm{~d}, J=8.69 \mathrm{~Hz}, 2 \mathrm{H}), 7.06(\mathrm{~d}, J=8.64 \mathrm{~Hz}, 2 \mathrm{H}), 7.36(\mathrm{~d}, J=8.66 \mathrm{~Hz}, 2 \mathrm{H}), 7.81(\mathrm{~d}, J=$ $8.42 \mathrm{~Hz}, 2 \mathrm{H}) .{ }^{13} \mathrm{C} \mathrm{NMR}\left(75 \mathrm{MHz}, \mathrm{CDCl}_{3}\right): \delta 12.4,12.7,21.5,25.4,34.7,42.5,52.5,62.9,107.8$, $114.0,126.4,128.3,128.6,129.6,130.2,146.6,158.9,163.7,169.5$. IR (chloroform): 2962 (w), 
2360 (m), 1716 (s), 1635 (s), 1540 (m), 1457 (m), 1196 (m), 1089 (m), 815 (m). HRMS $\left(\mathrm{CI}+\mathrm{CH}_{4}\right)$ Calcd for $\left[\mathrm{C}_{23} \mathrm{H}_{27} \mathrm{NO}_{5} \mathrm{~S}+\mathrm{H}^{+}\right]: 430.1689$; Found. $[\mathrm{M}+\mathrm{H}]^{+}: 430.1676$.

(S)-2-sec-Butyl-1-(3,4-dimethoxybenzyl)-5-oxo-2,5-dihydro-1H-pyrrol-3-yl phenylacetate 7f

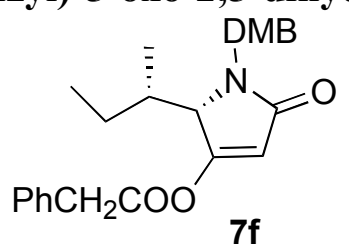

(Procedure D). Compound $2 \mathbf{f}(0.12 \mathrm{~g}, 0.12 \mathrm{mmol})$ gave $7 \mathbf{f}(75 \mathrm{mg}, 0.18 \mathrm{mmol}, 73 \%)$. $[\alpha]_{\mathrm{D}}^{19.8}-22$ $\left(c 0.38, \mathrm{CHCl}_{3}\right) .{ }^{1} \mathrm{H} \mathrm{NMR}\left(300 \mathrm{MHz}, \mathrm{CDCl}_{3}\right): \delta 0.89(\mathrm{~m}, 6 \mathrm{H}), 1.48(\mathrm{~m}, 2 \mathrm{H}), 1.93(\mathrm{~m}, 1 \mathrm{H}), 3.01$ (s, $2 \mathrm{H}), 3.64$ (s, $2 \mathrm{H}), 3.70(\mathrm{~d}, J=3.04 \mathrm{~Hz}, 1 \mathrm{H}), 3.85$ (s, $3 \mathrm{H}), 3.87$ (s, $3 \mathrm{H}), 3.94$ (s. 1H), 5.25 $(\mathrm{d}, J=14.64 \mathrm{~Hz}, 1 \mathrm{H}), 6.69-6.83(\mathrm{~m}, 3 \mathrm{H}), 7.26-7.35(\mathrm{~m}, 5 \mathrm{H}) .{ }^{13} \mathrm{C} \mathrm{NMR}\left(75 \mathrm{MHz}, \mathrm{CDCl}_{3}\right): \delta$ $12.1,13.5,25.1,35.2,40.9,42.5,43.3,55.9,68.5,111.1,111.5,120.9,127.2,127.6,128.6$, 129.3, 133.4, 148.9, 149.4, 161.8, 169.3, 206.3. IR ( $\left.\mathrm{CDCl}_{3}\right): 2962$ (w), 2360 (s), 1717 (s), 1698 (s), $1652(\mathrm{~m}), 1540(\mathrm{~m}), 1456(\mathrm{~m}), 1261(\mathrm{~m}), 1027(\mathrm{~m}), 810(\mathrm{~m}) \mathrm{cm}^{-1}$. HRMS (CI+CH4) Calcd for $\mathrm{C}_{17} \mathrm{H}_{23} \mathrm{NO}_{4}\left[\mathrm{M}+\mathrm{H}-\mathrm{PhCH}_{2} \mathrm{CO}\right]^{+}:$305.1627; Found. 305.1598 .

\section{(S)-1-Benzyl-2-sec-butyl-5-oxo-2,5-dihydro-1H-pyrrol-3-yl benzoate $7 \mathrm{~g}$}

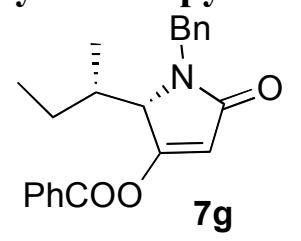

(Procedure D). Isoleucine derivative $2 \mathrm{~g}(0.22 \mathrm{~g}, 0.63 \mathrm{mmol})$ gave $7 \mathrm{~g}(0.16 \mathrm{~g}, 0.46 \mathrm{mmol}, 73 \%)$ as a colorless oil. $[\alpha]^{21}{ }_{\mathrm{D}}-14\left(c 2.2, \mathrm{CHCl}_{3}\right) .{ }^{1} \mathrm{H} \mathrm{NMR}\left(300 \mathrm{MHz}, \mathrm{CDCl}_{3}\right): \delta 0.81(\mathrm{~d}, J=6.92 \mathrm{~Hz}$, $3 \mathrm{H}), 0.93(\mathrm{t}, J=7.40 \mathrm{~Hz}, 3 \mathrm{H}), 1.12(\mathrm{~m}, 1 \mathrm{H}), 1.58(\mathrm{~m}, 1 \mathrm{H}), 2.01(\mathrm{~m}, 1 \mathrm{H}), 3.76(\mathrm{~d}, J=15.39$ $\mathrm{Hz}, 1 \mathrm{H}), 4.06(\mathrm{~d}, J=2.60 \mathrm{~Hz}, 1 \mathrm{H}), 5.30(\mathrm{~d}, J=15.37 \mathrm{~Hz}, 1 \mathrm{H}), 6.36(\mathrm{~s}, 1 \mathrm{H}), 7.65(\mathrm{~m}, 10 \mathrm{H})$. ${ }^{13} \mathrm{C}$ NMR $\left(75 \mathrm{MHz}, \mathrm{CDCl}_{3}\right): \delta 12.5,12.9,26.0,34.8,43.1,62.9,108.8,127.5,127.9,128.7$, 128.8, 129.9, 133.2, 134.3, 136.9, 162.0, 164.1, $171.2 \mathrm{~cm}^{-1} . \mathrm{IR}\left(\mathrm{CDCl}_{3}\right): 2964(\mathrm{w}), 2360(\mathrm{~s})$, 1757 (s), 1716 (s), 1653 (m), 1455 (m), 1237 (m), 1173 (m), 1043 (m), 844 (m), 704 (m). HRMS $\left(\mathrm{CI}+\mathrm{CH}_{4}\right)$ Calcd for $\left[\mathrm{C}_{22} \mathrm{H}_{23} \mathrm{NO}_{3}+\mathrm{H}^{+}\right]: 350.1756$; Found. $[\mathrm{M}+\mathrm{H}]^{+}: 350.1736$.

\section{(S)-1,5-Dibenzyl-4-(tert-butyldiphenylsilyloxy)-3-methyl-1 $H$-pyrrol-2(5H)-one 7 i}

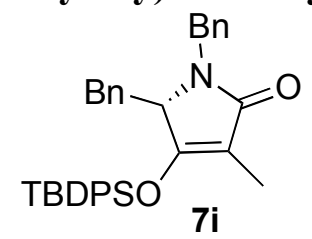

(Procedure C). Compound $2 \mathbf{i}(0.34 \mathrm{~g}, 0.84 \mathrm{mmol})$ gave $7 \mathbf{i}(0.35 \mathrm{~g}, 0.65 \mathrm{mmol}, 77 \%)$ as a colorless oil. $[\alpha]^{23} \mathrm{D}-31\left(c\right.$ 1.75, $\left.\mathrm{CDCl}_{3}\right){ }^{1} \mathrm{H}$ NMR (300 MHz, $\left.\mathrm{CDCl}_{3}\right): \delta 1.08(\mathrm{~s}, 9 \mathrm{H}), 1.30(\mathrm{~s}, 3$ H), $2.72(\mathrm{dd}, J=14.4 \mathrm{~Hz}, J=6.6 \mathrm{~Hz}, 1 \mathrm{H}), 3.15(\mathrm{dd}, J=14.4 \mathrm{~Hz}, J=3.3 \mathrm{~Hz}, 1 \mathrm{H}), 3.64$ (d, 
$J=15.3 \mathrm{~Hz}, 1 \mathrm{H}), 3.74(\mathrm{~m}, 1 \mathrm{H}), 5.17(\mathrm{~d}, J=15.3 \mathrm{~Hz}, 1 \mathrm{H}), 6.80-7.65(\mathrm{~m}, 20 \mathrm{H}) .{ }^{13} \mathrm{C}$ NMR $(75$ $\left.\mathrm{MHz}, \mathrm{CDCl}_{3}\right): \delta 8.3,20.1,27.0,36.8,44.5,60.3,110.3,127.3,127.6,128.2,128.5,128.8,128.9$, $129.8,131.0,131.1,132.3,132.6,135.6,135.8,136.8,138.1,164.5,173.8$. IR (thin film): 3070 (w), 3030 (w), 2931 (w), 2859 (w), 1677 (s), 1428 (w), 1389 (m), 1339 (m), 1225 (w), 1114 (m), $734(\mathrm{~m}), 700(\mathrm{~s}) \mathrm{cm}^{-1}$. Anal. Calcd for $\mathrm{C}_{35} \mathrm{H}_{37} \mathrm{NO}_{2} \mathrm{Si}: \mathrm{C}, 79.03 ; \mathrm{H}, 7.03 ; \mathrm{N}, 2.63$. Found: C, 78.76; H, 7.18; N, 2.63.

\section{(S)-1-Benzyl-4-(tert-butyldiphenylsilyloxy)-5-isobutyl-3-methyl-1H-pyrrol-2(5H)-one 7j}

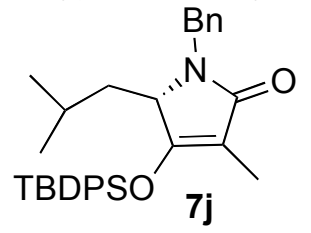

(Procedure C). Compound $\mathbf{2 j}(0.065 \mathrm{~g}, 0.176 \mathrm{mmol})$ gave $7 \mathbf{j}(0.083 \mathrm{~g}, 0.16 \mathrm{mmol}, 95 \%)$ as a colorless oil. $[\alpha]^{24.7}-45\left(c 0.44, \mathrm{CHCl}_{3}\right){ }^{1} \mathrm{H}$ NMR (300 MHz, $\left.\mathrm{CDCl}_{3}\right): \delta 0.76(\mathrm{~d}, J=6.3 \mathrm{~Hz}, 3$ $\mathrm{H}), 0.85(\mathrm{~d}, J=6.3 \mathrm{~Hz}, 3 \mathrm{H}), 1.05(\mathrm{~s}, 9 \mathrm{H}), 1.32$ (s, $3 \mathrm{H}), 1.59(\mathrm{~m}, 3 \mathrm{H}), 3.55$ (br s, $1 \mathrm{H}), 3.94$ (d, $J=15.6 \mathrm{~Hz}, 1 \mathrm{H}), 5.16(\mathrm{~d}, J=15.6 \mathrm{~Hz}, 1 \mathrm{H}), 7.08(\mathrm{~m}, 2 \mathrm{H}), 7.20-7.73(\mathrm{~m}, 13 \mathrm{H}) .{ }^{13} \mathrm{C}$ NMR $(75$ $\left.\mathrm{MHz}, \mathrm{CDCl}_{3}\right): \delta 8.3,20.0,23.5,24.2,24.5,26.8,27.0,38.2,44.2,58.9,109.4,127.5,127.9$, $128.03,128.3,128.4,129.0,129.9,130.8,130.9,132.3,132.6,135.3,135.5,135.6,138.3,165.4$, 173.7. IR (thin film): 2957 (s), 2932 (s), 2861 (m), 1686 (s), 1619 (s), 1429 (m), 1405 (m), 1339 (s), 1220 (s), 1115 (s), $842(\mathrm{~m}), 822$ (m), 772 (m), 734 (s), 701 (s) cm $\mathrm{cm}^{-1}$. Anal. Calcd for $\mathrm{C}_{32} \mathrm{H}_{39} \mathrm{NO}_{2} \mathrm{Si}$ : C, 77.22; H, 7.91; N, 2.81. Found: C, 76.92; H, 7.88; N, 2.45.

\section{Racemization of $t$-butyldiphenylsilyl enol ethers of tetramic acids ( $r a c 7 a, b)$}

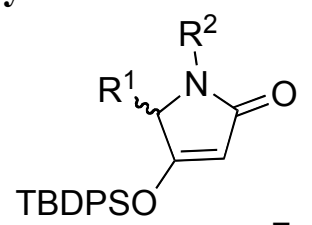

rac. $7 a, b$

7a: $R^{1}=B n, R^{2}=B n$

7b: $\mathrm{R}^{1}=i-\mathrm{Bu}, \mathrm{R}^{2}=\mathrm{Bn}$

Lithium telluride $(0.45 \mathrm{~mL}, 0.11 \mathrm{mmol}, 0.25 \mathrm{M}$ suspension in THF) was added to a solution of haloacylamino acid esters $\mathbf{2 a}, \mathbf{2 b}(0.11 \mathrm{mmol})$ in THF $(1.0 \mathrm{~mL})$ followed by addition of LHMDS $(0.11 \mathrm{~mL}, 0.11 \mathrm{mmol}, 1 \mathrm{M})$ at room temperature. The mixture was stirred for $40 \mathrm{~min}$, and freshly distilled $t-\mathrm{BuPh}_{2} \mathrm{SiCl}$ (3.5 equiv.) was added by syringe. Stirring was continued for $20 \mathrm{~h}$. The reaction was quenched by addition of saturated aqueous $\mathrm{NH}_{4} \mathrm{Cl}(1 \mathrm{~mL})$, and the elemental tellurium was removed by passing the mixture through a short column of celite. Extraction with ethyl acetate $(3 \times 2 \mathrm{~mL})$, drying $\left(\mathrm{Na}_{2} \mathrm{SO}_{4}\right)$, and chromatography on silica gel (hexane/EtOAc $3: 1$ ) gave either $7 \mathbf{a}(52 \mathrm{mg}, 0.10 \mathrm{mmol}, 92 \%)$ or $7 \mathbf{b}(53 \mathrm{mg}, 0.11 \mathrm{mmol}, 100 \%)$ as a colorless oils. The phenylalanine derivative 7a was subjected to HPLC [Chiralcel AS-H column, eluent $i$ $\mathrm{PrOH} /$ hexane 15:85, $1 \mathrm{~mL} / \mathrm{min}$, UV detector at $254 \mathrm{~nm}$ ] which revealed racemization. 
Enantiomeric ratio: 53:47, retention times, 26.3 and $40.5 \mathrm{~min}$. The ${ }^{1} \mathrm{H}$ NMR spectrum of the TBDPS enol ether of the crude tetramic acid derived from $L$-isoleucine showed a doublet of doublets at $\delta 3.82$ and $\delta 3.85 \mathrm{ppm}$ in nearly equal intensity for the C-5 proton (the $\alpha$-proton of the amino acid precursor) which indicates complete or nearly complete racemization with 3.5 equivalents of TBDPSCl as compared to a lone doublet at $\delta 3.84$ ppm with 1.15 equivalents of TBDPSCl.

$(4 S, 5 S)-5-B e n z y l-4-h y d r o x y-1-(4-m e t h o x y b e n z y l) p y r r o l i d i n-2-o n e ~ 8^{25}$

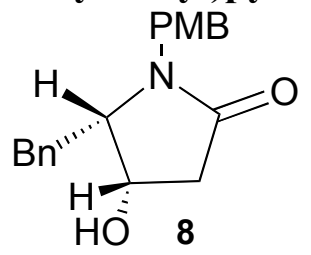

To a stirred solution of tetramic acid $6 \mathbf{c}$ (prepared according to procedure $\mathrm{B}$ ) (0.6 g, $1.93 \mathrm{mmol}$ ) in $\mathrm{CH}_{2} \mathrm{Cl}_{2}$ :AcOH (10:1) was added $\mathrm{NaBH}_{4}(0.15 \mathrm{~g}, 3.7 \mathrm{mmol})$ and the reaction mixture was stirred at $0{ }^{\circ} \mathrm{C}$ for $4 \mathrm{~h}$. The reaction mixture was monitored by TLC and quenched with water. Extraction with methylene chloride $(3 \times 10 \mathrm{~mL})$, drying $\left(\mathrm{Na}_{2} \mathrm{SO}_{4}\right)$ and concentration gave the crude product, which was purified by column chromatography (EtOAc:hexane, 2:1) to afford 8 $(0.52 \mathrm{~g}, 1.67 \mathrm{mmol}, 87 \%)$ as a white solid: m.p. $145^{\circ} \mathrm{C}$. [lit. ${ }^{25 a, b}$ m.p. $\left.144.5-145.5{ }^{\circ} \mathrm{C}\right] \cdot[\alpha]^{22}{ }_{\mathrm{D}}-78$ $\left.(c), \mathrm{CHCl}_{3}\right)$. [lit. ${ }^{25 \mathrm{a}}[\alpha]^{20} \mathrm{D}-75\left(c 0.7, \mathrm{CHCl}_{3}\right)$.]. ${ }^{1} \mathrm{H} \mathrm{NMR}\left(\mathrm{CDCl}_{3}, 500 \mathrm{MHz}\right): \delta 2.16(\mathrm{~d}, J=5.41$ $\mathrm{Hz}, 1 \mathrm{H}), 2.43(\mathrm{dd}, J=17.09,3.28 \mathrm{~Hz}, 1 \mathrm{H}), 2.59(\mathrm{dd}, J=17.16,6.35 \mathrm{~Hz}, 1 \mathrm{H}), 3.0(\mathrm{~d}, J=7.52$ Hz, 2 H), $3.67(\mathrm{~m}, 1 \mathrm{H}), 3.80(\mathrm{~s}, 3 \mathrm{H}), 3.83(\mathrm{~d}, J=14.96 \mathrm{~Hz}, 1 \mathrm{H}), 4.17(\mathrm{~m}, 1 \mathrm{H}), 5.02(\mathrm{~d}, J=$ $14.91 \mathrm{~Hz}, 1 \mathrm{H}), 6.85(\mathrm{~d}, J=8.68 \mathrm{~Hz}, 2 \mathrm{H}), 7.09(\mathrm{~d}, J=8.63 \mathrm{~Hz}, 2 \mathrm{H}), 7.14(\mathrm{~d}, J=7.18 \mathrm{~Hz}, 2 \mathrm{H})$, $7.21(\mathrm{~m}, 1 \mathrm{H}), 7.27(\mathrm{~d}, J=6.92 \mathrm{~Hz}, 2 \mathrm{H}) .{ }^{13} \mathrm{C} \mathrm{NMR}\left(\mathrm{CDCl}_{3}, 125 \mathrm{MHz}\right): \delta 32.8,40.3,43.5,55.2$, $62.9,65.9,114.0,126.5,128.3,128.5,129.1,129.2,137.5,158.9,173.5$. IR (Chloroform): 3422 (s), $2957(\mathrm{~s}), 2360$ (s), 1698 (s), $1558(\mathrm{~m}), 820(\mathrm{~m}), 700(\mathrm{~m}), 668(\mathrm{~m}) \mathrm{cm}^{-1}$.

\section{Acknowledgements}

This research was supported by Syracuse University. Matthew Purzycki, Zachary Martens, Elliot Hohn, and Matthew Bacler were supported by the NSF-Research Experience for Undergraduates program. We thank Professor John Chisholm for the use of an HPLC instrument and Patrick Carberry for his help.

\section{References and Notes}

1. Preliminary reports: (a) Kandula, V. S.; Avilov, D.; Carminucci, A.; Purzycki, M.; Dittmer, D. C. Abstracts of Papers; $37^{\text {th }}$ Northeast Regional Meeting of the American Chemical 
Society, Burlington, VT; American Chemical Society: Washington, DC, 2008; NERM 289. (b) Dittmer, D. C.; Avilov, D. V.; Purzycki, M. T. Abstracts of Papers; $234^{\text {th }}$ National Meeting of the American Chemical Society, Boston, MA; American Chemical Society: Washington, DC, 2007; ORGN 1003. (c) Dittmer, D. C.; Avilov, D. V.; Hohn, E. B.; Martens, Z. J.; Li, Q.; Bacler, M. W. Abstracts of Papers; $232^{\text {nd }}$ National Meeting of the American Chemical Society, San Francisco, CA; American Chemical Society: Washington, DC, 2006; ORGN 529. (d) Dittmer, D. C.; Avilov, D. V.; Purzycki, M. T.; Martens, Z. J.; Hohn, E. B.; Li, Q.; Bacler, M. W. Abstracts of Papers; $35^{\text {th }}$ Northeast Regional Meeting of the American Chemical Society, Binghamton, NY; American Chemical Society: Washington, DC, 2006; NRM 141.

2. Reviews: (a) Royles, B. L. J. Chem. Rev.1995, 95, 1981. (b) Schobert, R.; Schlenk, A. Bioorg. Med. Chem. 2008, 16, 4203. (c) Ghisalberti, E. L. Studies Nat. Products Chem. 2003, 28, 109. (d) Gossauer, A. Proc. Chem. Org. Nat. Prod. 2003, 86, 1. (e) Huang, P-Q. Synlett 2006, 1133. (f) Shimshock, S. J.; DeShong, P. Studies Nat. Products Chem. 1994, 14, 97. (g) Henning, H-G.; Gelbin, A. Adv. Heterocycl. Chem. 1993, 57, 139. (h) Markopoulos, J.; Detsi, A.; Gavrielatos, E.; Athanasellis, G.; Igglessi-Markopoulou, O. Biomed. Health Res. 2002, 55, 25. (i) Cox, R. J. In Symposium of the British Mycological Society 26 (Exploitation of Fungi); Robson, G. D., vanWest, P., Gadd, G. M., Eds., Cambridge University Press, 2007; pp 25. (j) Ivanov, A. S. Chem. Soc. Rev. 2008, 37, 789.

3. Kaufmann, G. F.; Sartorio, R.; Lee, S-H.; Rogers, C. J.; Meijler, M. M.; Moss, J. A.; Clapham, B.; Brogan, A. P.; Dickerson, T. J.; Janda, K. D. Proc. Natl. Acad. Sci. U.S.A. 2005, 102, 309.

4. (a) Kürti, L.; Czakó, B. Strategic Applications of Named Reactions in Organic Synthesis, Elsevier Academic Press: Burlington, MA, 2007, p 138. (b) 374. (c) Halo, L. M.; Marshall. J. W.; Yakasai, A. A.; Song, Z.; Butts, C. P.; Crump, M. P.; Heneghan, M.; Bailey, A. M.; Simpson, T. J.; Lazarus, C. M.; Cox, R. J. Chem.Bio.Chem 2008, 9, 585. (d) Sims, J. W.; Schmidt, E. W. J. Am. Chem. Soc. 2008, 130, 11149.

5. (a) Büchi, G.; Lukas, G. J. Am. Chem. Soc. 1964, 86, 5654. (b) Poncet, J.; Jouin, P.; Castro, B.; Nicolas, L.; Boutar, M.; Gaudemer, A. J. Chem. Soc., Perkin Trans 1 1990, 611.

6. Ley, S. V.; Smith, S. C.; Woodward, P. R. Tetrahedron Lett. 1988, 29, 5829.

7. Ley, S. V.; Smith, S. C.; Woodward, P. R. Tetrahedron 1992, 48, 1145.

8. Klutchko, S.; O’Brien, P.; Hodges, J. C. Synth. Commun. 1989, 19, 2573.

9. Fitch, D. M.; Evans, K. A.; Chai, D.; Duffy, K. J. Org. Lett. 2005, 7, 5521.

10. Matthews, J.; Rivero, R.A.; J. Org. Chem. 1998, 63, 4808.

11. Andrews, M. D.; Brewster, A. G.; Crapnell, K. M.; Ibbett, A. J.; Jones, T.; Moloney, M. G.; Prout, K.; Watkin, D. J. Chem. Soc., Perkin Trans 1 1998, 223.

12. (a) Mulholland, T. P. C.; Foster, R.; Haydock, D. B. J. Chem. Soc., Perkin Trans. 1 1972, 2121. (b) Holzapfel, C. W.; Kruger, F. W. H. Aust. J. Chem. 1992, 45, 97.

13. (a) Kulkarni, B. A.; Ganesan, A. Tetrahedron Lett. 1998, 39, 4369. (b) Kulkarni, B. A.; Ganesan, A. Angew. Chem. Int. Ed. 1997, 36, 2454. (c) Evans, K. A.; Chai, D.; Graybill, T. 
L.; Burton, G.; Sarisky, R. T.; Lin-Goerke, J.; Johnson, V. K.; Rivero, R. A. Bioorg. Med. Chem. Lett. 2006, 16, 2205. (d) Larbig, G.; Schmidt, B. J. Comb. Chem. 2006, 8, 480. (e) Prousis, K. C.; Detsi, A.; Igglessi-Markopoulou, O. Synlett 2005, 2763.

14. Kruger, P. E. J.; Arndt, R. R. J. S. Afr. Chem. Inst. 1973, 26, 132.

15. van der Baan, J. L.; Barnick, J. W. F. K.; Bickelhaupt, F. Tetrahedron 1978, 34, 223.

16. Heinicke, G. W.; Morella, A. M.; Orban, J.; Prager, R. H.; Ward, A. D. Aust. J. Chem. 1985, $38,1847$.

17. Bergson, G. Acta Chem. Scand. 1957, 11, 571.

18. Clive, D. L. J.; Beaulieu, P. L. J. Org. Chem. 1982, 47, 1124.

19. Engman, L.; Cava, M. P. J. Org. Chem. 1982, 47, 3946.

20. Osuka, A.; Suzuki, H. Chem. Lett. 1983, 119.

21. Dittmer, D. C.; Li, Q.; Avilov, D. V. J. Org. Chem. 2005, 70, 4682 and references cited therein.

22. Some reactions are reviewed: Petragnani, N.; Stefani, H. Tellurium In Organic Synthesis, $2^{\text {nd }}$ Ed.; Academic Press: London, 2007; pp 147.

23. (a) Oguz, U.; Guilbeau, G. G.; McLaughlin, M. L. Tetrahedron. Lett. 2002, 43, 2873. (b) Bonache, M. A.; Gerona-Navarro, G.; Garcia-Aparicio, C.; Alias, M.; Martin-Martinez, M.; Garcia-Lopez, M. T.; López, P.; Cativiela, C.; González-Muniz, R. Tetrahedron: Asymmetry 2003, 14, 2161.

24. Odysseus had to pass between the monster Scylla on the one side and the whirlpool Charybdis on the other to successfully navigate the Strait of Messina: Lawrence, T. E., The Odyssey of Homer (New English Prose Translation) Oxford: New York, 1991; Book XII, p 171.

25. (a) Huang, P-Q.; Wu, T-J.; Ruan, Y-P. Org. Lett. 2003, 5, 4341. (b) Galeotti, N.; Poncet, J.; Chiche, L.; Jouin, P. J. Org. Chem. 1993, 58, 5370. (c) Murray, A.; Proctor, G. R.; Murray, P. J. Tetrahedron 1996, 52, 3757.

26. (a) Nahm, S.; Weinreb, S. M. Tetrahedron Lett. 1981, 22, 3815. (b) Gibson, C. L.; Kennedy, A. R.; Morthala, R. R.; Parkinson, J. A.; Suckling, C. J. Tetrahedron 2008, 64, 7619.

27. (a) Bosshard, H. H.; Mory, R.; Schmid, M.; Zollinger, H. Helv. Chim. Acta 1959, 42, 1653. (b) Barluenga, J.; Campos, P. J.; Gonzalez-Nuñez, E.; Asensio, G. Synthesis 1985, 426.

28. Katsuki, T.; Yamaguchi, M. Bull. Chem. Soc. Jpn. 1976, 49, 3287.

29. (a) Nagayama, S.; Kobayashi, S. J. Am. Chem. Soc. 2000, 122, 11531. (b) Kobayashi, S.; Manabe, K. Acc, Chem. Res. 2002, 35, 209. (c) Li. H-J.; Tian, H-Y.; Wu, Y-C.; Chen, Y-J.; Liu, L.; Wang, D.; Li, C-J. Adv. Synth. Catal. 2005, 347, 1247.

30. (a) Ranganathan, D., Farooqui, F.; Mehrota, S.; Kesavan, K. Heterocycles 1986, 24, 2493.

(b) Lambert, T. H.; Danishevsky, S. J. J. Am. Chem. Soc. 2006, 128, 426.

31. Wuts, P. G. M.; Greene, T. W., Greene's Protective Groups In Organic Synthesis, $4^{\text {th }}$ Ed.; Wiley-Interscience: Hoboken, NJ, 2007, p 894.

32. Li, W-R.; Lin, S. T.; Hsu, N-M.; Chern, M-S. J. Org. Chem 2002, 67, 4702.

33. Murray, A.; Proctor, G. R. Tetrahedron 1995, 36, 291. 